\title{
Nanospintronics with carbon nanotubes
}

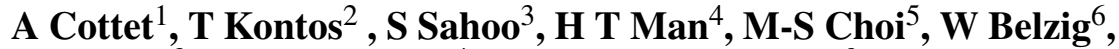 \\ C Bruder $^{3}$, A F Morpurgo ${ }^{4}$ and C Schönenberger ${ }^{3}$ \\ ${ }^{1}$ Laboratoire de Physique des Solides, Bât. 510, Université Paris Sud, 91405 Orsay Cedex, \\ France \\ ${ }^{2}$ Laboratoire Pierre Aigrain, Ecole Normale Supérieure, 24 rue Lhomond, \\ 75231 Paris Cedex 05, France \\ ${ }^{3}$ Institute of Physics, University of Basel, Klingelbergstrasse 82, CH-4056 Basel, Switzerland \\ ${ }^{4}$ Kavli Institute of NanoScience Delft, Faculty of Applied Sciences, Delft University of \\ Technology, Lorentzweg 1, 2628 CJ Delft, The Netherlands \\ ${ }^{5}$ Department of Physics, Korea University, Seoul 136-701, Korea \\ ${ }^{6}$ Department of Physics, University of Konstanz, M703, D-78457 Konstanz, Germany \\ E-mail: Audrey.Cottet@lps.u-psud.fr and kontos@lpa.ens.fr
}

Received 5 June 2006, in final form 27 July 2006

Published 17 October 2006

Online at stacks.iop.org/SST/21/S78

\begin{abstract}
One of the actual challenges of spintronics is the realization of a spin transistor allowing control of spin transport through an electrostatic gate. In this paper, we report on different experiments which demonstrate gate control of spin transport in a carbon nanotube connected to ferromagnetic leads. We also discuss some theoretical approaches which can be used to analyse spin transport in these systems. We emphasize the roles of the gate-tunable quasi-bound states inside the nanotube and the coherent spin-dependent scattering at the interfaces between the nanotube and its ferromagnetic contacts.
\end{abstract}

(Some figures in this article are in colour only in the electronic version)

\section{Introduction: nanospintronics}

The quantum mechanical spin degree of freedom is now widely exploited to control current transport in electronic devices. For instance, the readout of magnetic hard discs is based on the spin-valve effect, i.e. the tunability of a conductance through the relative orientation of some ferromagnetic polarizations [1]. However, realizing spin injection in nanostructures, e.g. mesoscopic conductors or molecules, would allow us to implement further functionalities. For example, the realization of a 'spin transistor' would allow electric field control of the spin-valve effect through an electrostatic gate $[2,3]$. In this context, carbon nanotubes are particularly interesting, because they should exhibit a long spin lifetime and can be contacted with ferromagnetic materials. In this paper, we present the state of the art regarding the realization of spin-transistor-like devices with carbon nanotubes. In section 2, we introduce the basics of the spin-valve effect. In section 3, we present a theoretical description of spin transport in quantum wires with ferromagnetic contacts. We put special emphasis on the roles of the gate-tunable resonant states inside the wire and the coherent spin-dependent scattering at the boundaries of the wire. In section 4, we present the state of the art in contacting carbon nanotubes with ferromagnetic materials and evoke different contact effects which could mimic spindependent transport phenomena. In section 5, we review different experiments which have demonstrated gate control of spin transport in carbon nanotubes so far. Eventually, we give some conclusions and perspectives in section 6 .

\section{The spin-valve geometry}

The most standard method to inject or detect spins in an insulating or conducting element $M$ is to use the spinvalve geometry $[4,5]$, in which $\mathrm{M}$ is connected to two ferromagnetic leads $\mathrm{L}$ and $\mathrm{R}$ (figure 1, left). One has to measure the conductances $G^{\mathrm{P}}$ and $G^{\mathrm{AP}}$ of the spin valve for lead magnetizations in the parallel (P) and antiparallel (AP) configurations. This requires the use of two ferromagnets with different coercive fields ( $H_{\mathrm{cL}}$ and $H_{\mathrm{cR}}$, respectively) for switching one magnetization with respect to the other with the help of an external magnetic field $H$ (figure 1, right). The spin signal or magnetoresistance is then defined as the relative difference $\mathrm{MR}=\left(G^{\mathrm{P}}-G^{\mathrm{AP}}\right) / G^{\mathrm{AP}}$. 

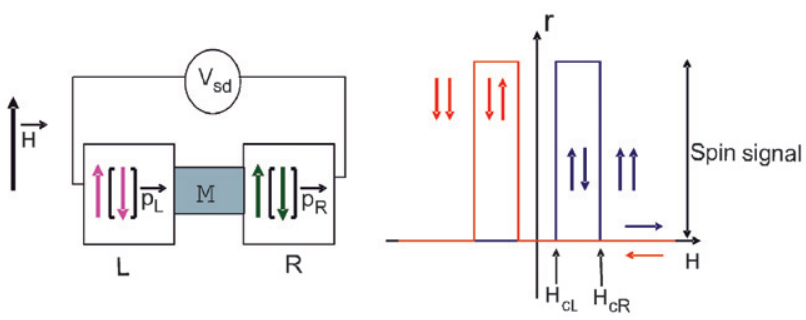

Figure 1. Left: electrical diagram of a circuit with the spin-valve geometry. The element $\mathrm{M}$ is connected to two ferromagnetic leads $\mathrm{L}$ and $\mathrm{R}$, in which the electronic density of states has magnetic polarizations $\vec{p}_{\mathrm{L}}$ and $\vec{p}_{\mathrm{R}}$. The three elements in series form a spin valve, which is voltage biased with a source-drain voltage $V_{\text {sd }}$. A magnetic field $\vec{H}$ is applied to the circuit. Right: typical shape of the resistance curve $r(H)$ measured in the spin valve while increasing (blue (dark grey) line) and then decreasing (red (light grey) line) $H$. Since the two contacts $\mathrm{L}$ and $\mathrm{R}$ have different coercive fields $H_{\mathrm{cL}}$ and $H_{\mathrm{cR}}$, it is possible to selectively reverse the directions of $\vec{p}_{\mathrm{L}}$ and $\vec{p}_{\mathrm{R}}$ during this cycle. This introduces a hysteretic pattern in the $r(H)$ curve, from which the value of the magnetoresistance $\mathrm{MR}=\left(G^{\mathrm{P}}-G^{\mathrm{AP}}\right) / G^{\mathrm{AP}}$ of the spin valve can be obtained. Here, one has $\mathrm{MR}>0$.

Let us consider the situation in which the element placed between the two ferromagnetic contacts is a tunnelling barrier with a transmission probability independent of energy [6]. This case, usually referred to as Jullière's model, describes the principle of magnetic memories and magnetic read heads. From Fermi's golden rule, the transmission probability of the barrier for spins $\sigma \in\{\uparrow, \downarrow\}$ is proportional to the electronic densities of states at the Fermi energy $N_{l, \sigma}=N_{l}\left(1+\sigma \eta_{l} p_{l}\right)$ for spins $\sigma$ at both contacts, with $l \in\{\mathrm{L}, \mathrm{R}\}$ and $\eta_{l} \in$ $\{+1,-1\}$ the direction of the magnetization at contact $l$. Here, $N_{l}$ is the spin-averaged density of states and $p_{l}$ is the spin polarization at contact $l$. The conductance $G^{\mathrm{P}}$ of the barrier in the parallel configuration is proportional to $N_{\mathrm{L}} N_{\mathrm{R}}\left[\left(1+p_{\mathrm{L}}\right)\left(1+p_{\mathrm{R}}\right)+\left(1-p_{\mathrm{L}}\right)\left(1-p_{\mathrm{R}}\right)\right]$ whereas the conductance $G^{\mathrm{AP}}$ in the anti-parallel configuration is proportional to $N_{\mathrm{L}} N_{\mathrm{R}}\left[\left(1+p_{\mathrm{L}}\right)\left(1-p_{\mathrm{R}}\right)+\left(1-p_{\mathrm{L}}\right)\left(1+p_{\mathrm{R}}\right)\right]$. This leads to

$$
\mathrm{MR}=\frac{2 p_{\mathrm{L}} p_{\mathrm{R}}}{1-p_{\mathrm{L}} p_{\mathrm{R}}}
$$

If the spin polarizations $p_{\mathrm{L}}$ and $p_{\mathrm{R}}$ have the same sign, the magnetoresistance of the device is positive because the current flowing in the antiparallel configuration is lower due to the imbalance between $N_{\mathrm{L}, \sigma}$ and $N_{\mathrm{R}, \sigma}$.

In the following, we consider the case in which element $\mathrm{M}$ is a carbon nanotube. In contrast with Jullière's model of a tunnelling barrier, the transmission of the nanotube cannot be considered as constant with energy due to the existence of quasi-bound states between the two ferromagnetic contacts. Furthermore, it is possible to tune the energy of these bound states with an electrostatic gate. This significantly modifies the behaviour of carbon-nanotube-based spin valves, as we are going to explain theoretically in the next section.

\section{Spin transport in finite-size quantum wires}

Carbon nanotubes can display a large variety of behaviour, depending on their intrinsic properties and on the characteristics of their electrical contacts. Even in the case of a clean nanotube (i.e. with no structural defects), electronic

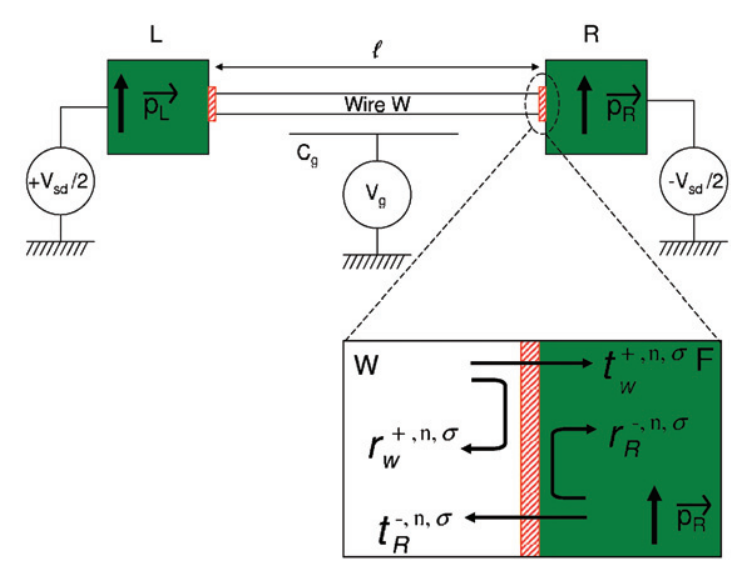

Figure 2. Electrical diagram of a ballistic wire $\mathrm{w}$ of length $\ell$ connected to ferromagnetic leads $\mathrm{L}$ and $\mathrm{R}$ with magnetic polarizations $\vec{p}_{\mathrm{L}}$ and $\vec{p}_{\mathrm{R}}$. The wire is voltage biased with a source-drain voltage $V_{\text {sd }}$ and capacitively coupled to a gate voltage source $V_{\mathrm{g}}$. Inset: scattering description of the interface between the wire and a contact. We use transmission and reflection amplitudes $t_{l}^{\epsilon, n, \sigma}$ and $r_{l}^{\epsilon, n, \sigma}$ for electrons with spin $\sigma$ of transverse channel $n$, incident from element $l \in\{\mathrm{L}, \mathrm{R}, \mathrm{w}\}$ with direction $\epsilon(\epsilon=+$ for right-going incident electrons and $\epsilon=-$ for left-going incident electrons).

transport can occur in different regimes, depending on the transparency of the contacts. For high contact resistances $R>h / e^{2}$, a nanotube can behave as a quantum dot, in which the Coulomb blockade determines the transport properties [7], whereas for low contact resistances $R<h / e^{2}$, transport is mainly determined by quantum interference [8]. Here, we will mainly consider these two situations. For simplicity, we will model the nanotube as a one-dimensional quantum wire.

\subsection{Spin-dependent transport in a non-interacting ballistic wire}

Although electron-electron interactions should be of primary importance in one-dimensional quantum wires, it is instructive to first consider a non-interacting picture. In addition, as we will see in section 5 , such a simplified picture captures the main features of some available experiments.

3.1.1. Transmission of a F-wire-F ballistic system. We consider the circuit of figure 2, with $\mathrm{w}$ a non-interacting ballistic wire of length $\ell$ contacted to two ferromagnetic leads $\mathrm{L}$ and $\mathrm{R}$. In the non-interacting limit, electronic transport through this device can be described using a scattering approach [9]. As represented in the bottom inset of figure 2, this description involves complex amplitudes of transmission and reflection $t_{l}^{\epsilon, n, \sigma}$ and $r_{l}^{\epsilon, n, \sigma}$ for electrons with spin $\sigma$ of transverse channel $n$, incident from element $l \in\{\mathrm{L}, \mathrm{R}, \mathrm{w}\}$ with direction $\epsilon(\epsilon=+$ for right-going incident electrons and $\epsilon=-$ for left-going incident electrons). Assuming that the different channels $n$ are not coupled by interfacial scattering, the behaviour of the device only depends on the transmission probabilities $T_{\mathrm{L}(\mathrm{R})}^{n, \sigma}=\left|t_{\mathrm{L}(\mathrm{R})}^{+(-), n, \sigma}\right|^{2}$ and on the reflection phases $\varphi_{\mathrm{L}}^{n, \sigma}=\arg \left(r_{\mathrm{w}}^{-, n, \sigma}\right)$ and $\varphi_{\mathrm{R}}^{n, \sigma}=\arg \left(r_{\mathrm{w}}^{+, n, \sigma}\right)$ at the side of the wire. Indeed, the conductance of the circuit in configuration $\mathrm{P}[\mathrm{AP}]$ can be calculated from the expression

$$
G^{\mathrm{P}[\mathrm{AP}]}=G_{Q} \sum_{n, \sigma} \int_{-\infty}^{+\infty} \mathbb{T}_{\mathrm{P}[\mathrm{AP}]}^{n, \sigma}(E)(-\partial f(E) / \partial E),
$$



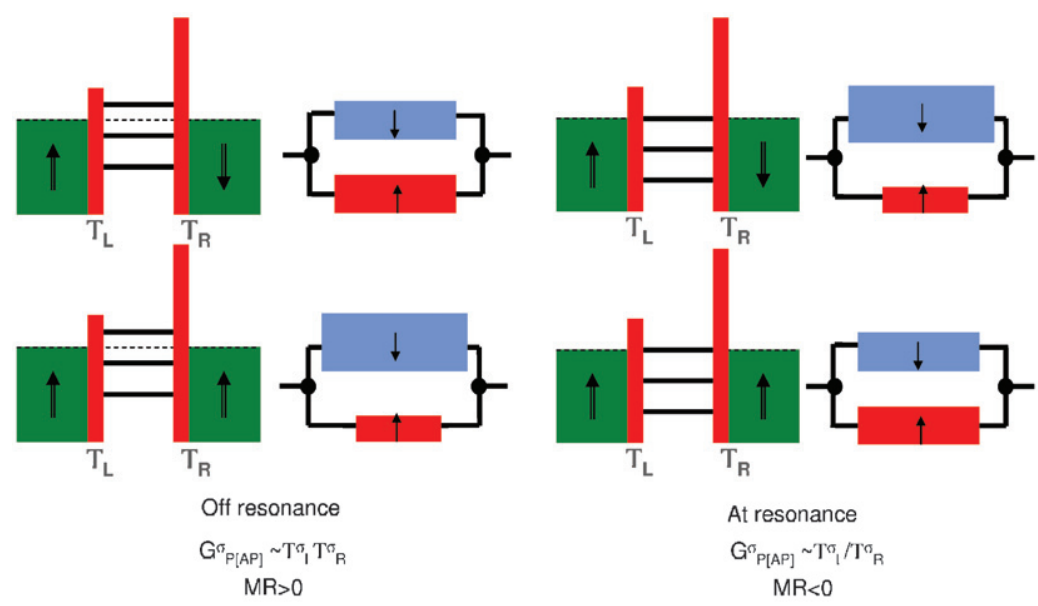

Figure 3. Picture of the resonant tunnelling mechanism for very asymmetric barriers. Here, we assume $T_{\mathrm{L}}^{\sigma} \ll T_{\mathrm{R}}^{\sigma}$ and $P_{\mathrm{L}}=P_{\mathrm{R}}$. A bigger resistance element represents a higher resistance value. Off resonance (left panels), the transmission probability $\mathbb{T}_{\mathrm{P}[\mathrm{AP}]}^{\sigma}$ of the circuit for spins $\sigma$ scales with $T_{\mathrm{L}}^{\sigma} T_{\mathrm{R}}^{\sigma}$ in the $\mathrm{P}[\mathrm{AP}]$ configuration. This leads to a positive MR like in Jullière's model. On resonance (right panels), $\mathbb{T}_{\mathrm{P}[\mathrm{AP}]}^{\sigma}$ scales with $4 T_{\mathrm{L}}^{\sigma} / T_{\mathrm{R}}^{\sigma}$, which leads to a negative $\mathrm{MR}$.

where $f(E)=\left[1+\exp \left(E / k_{\mathrm{B}} T\right)\right]^{-1}$ is the Fermi-Dirac distribution and where

$\mathbb{T}_{\mathrm{P}[\mathrm{AP}]}^{n, \sigma}=\frac{T_{\mathrm{L}}^{n, \sigma} T_{\mathrm{R}}^{n, \sigma}}{\mid 1-\left[\left(1-T_{\mathrm{L}}^{n, \sigma}\right)\left(1-T_{\mathrm{R}}^{n, \sigma}\right)\right]^{1 / 2} \mathrm{e}^{\left.\mathrm{i}\left(\varphi_{\mathrm{L}}^{n, \sigma}+\varphi_{\mathrm{R}}^{n, \sigma}+2 \delta_{0}+\sigma \gamma_{\mathrm{H}}^{n}\right)\right|^{2}}}$

is the probability that an electron of channel $n$ with spin $\sigma$ coming from lead $\mathrm{L}(\mathrm{R})$ is transmitted to lead $\mathrm{R}(\mathrm{L})$. Here, we have introduced the orbital phase $\delta_{0}=\ell k_{\mathrm{Fw}}^{n}\left(V_{\mathrm{g}}\right)$ acquired by an electron upon crossing the wire once and $\gamma_{\mathrm{H}}^{n}=g \mu_{\mathrm{B}} H \ell / \hbar v_{\mathrm{FW}}^{n}$, with $H$ the external magnetic field. We call $k_{\mathrm{FW}}^{n}\left(V_{\mathrm{g}}\right)$ the gate-dependent wave vector of electrons of channel $n$ inside the wire, $v_{\mathrm{Fw}}^{n}\left(V_{\mathrm{g}}\right)$ the corresponding Fermi velocity, $g$ the Landé factor and $\mu_{\mathrm{B}}$ the Bohr magneton. The denominator of equation (2) accounts for the existence of resonant states which are due to multiple reflections between the two contacts. These resonances lead to peaks in the $G^{\mathrm{P}[\mathrm{AP}]}\left(V_{\mathrm{g}}\right)$ curves. In the case of ferromagnetic contacts, the interfacial scattering properties depend on spin and on the configuration $c \in\{\mathrm{P}, \mathrm{AP}\}$ of the ferromagnetic electrodes (we omit the index $c$ in $T_{\mathrm{L}(\mathrm{R})}^{n, \sigma}$ and $\varphi_{\mathrm{L}(\mathrm{R})}^{n, \sigma}$ for brevity). In the following, we investigate the effects of a finite spin polarization of the tunnelling rates $P_{l}^{n} \neq 0$ and of a spin dependence of interfacial phase shifts (SDIPS), i.e. $\Delta \varphi_{l}^{n} \neq 0$, with

$$
T_{l}^{n, \sigma}=T_{l}^{n}\left(1+\eta_{l} \sigma P_{l}^{n}\right), \quad \varphi_{l}^{n, \sigma}=\varphi_{l}^{n}+\eta_{l} \sigma \frac{\Delta \varphi_{l}^{n}}{2},
$$

for $l \in\{\mathrm{L}, \mathrm{R}\}$. Here, $\eta_{l} \in\{+1,-1\}$ denotes the direction of the magnetization at contact $l$.

The quantum wires which we have in mind are carbon nanotubes. Two different types of carbon nanotubes can be fabricated: single-wall nanotubes (SWNTs) and multi-wall nanotubes (MWNTs). A SWNT consists of a single graphene sheet that is rolled up into a cylinder. A MWNT consists of a set of coaxially stacked graphene cylinders. In the case of a SWNT, it is possible to have only two channels involved in current transport at low voltages (the energy levels of SWNTs often display a two-fold degeneracy related to the $K-K^{\prime}$ degeneracy of the energy bands of graphene $[10,11])$. Assuming two identical channels with no coupling, the behaviour of such a nanotube can be understood from the study of a one-channel quantum wire, which is presented in section 3.1.2 (the conductance of the nanotube will be twice that of the single-channel quantum wire and the magnetoresistance will be identical). For MWNTs, more channels are generally involved in the low-voltage electronic transport. We will thus present in section 3.1.3 the case of a quantum wire with several channels.

3.1.2. Single-channel case. In this section, we omit the channel index $n$. We assume that the gate voltage $V_{\mathrm{g}}$ induces a shift of the wire electrostatic potential which is small compared with the Fermi energy of the wire, i.e. $\alpha V_{\mathrm{g}} \ll E_{\mathrm{Fw}}$, where $\alpha=C_{\mathrm{g}} / C_{\Sigma}$ is the ratio between the gate capacitance and the total capacitance of the wire. In this limit, one finds $\delta_{0}=\ell k_{\mathrm{Fw}}+\left(e \alpha V_{\mathrm{g}}-E_{\mathrm{Fw}}\right)\left(\pi N_{\mathrm{Fw}} / 2\right)$ where $N_{\mathrm{Fw}}=2 \ell / \pi \hbar v_{\mathrm{Fw}}$ is the density of states in the wire, and $k_{\mathrm{Fw}}$ and $v_{\mathrm{Fw}}$ are the Fermi wave vector and velocity in the wire, respectively. Therefore, the resonant peaks in the $G^{\mathrm{P}[\mathrm{AP}]}\left(V_{\mathrm{g}}\right)$ curve correspond to the cancellation of resonant energies of the form

$$
E_{\mathrm{P}[\mathrm{AP}]}^{\sigma, j}=\left(2 \pi j-\varphi_{\mathrm{L}}^{\sigma}-\varphi_{\mathrm{R}}^{\sigma}-\sigma \gamma_{\mathrm{H}}\right)\left(\hbar v_{\mathrm{FW}} / 2 \ell\right)-e \alpha V_{\mathrm{g}},
$$

with $j \in \mathbb{Z}$.

Magnetoresistance of a one-channel wire with no SDIPS. In this paragraph, we investigate the behaviour of the wire for $\Delta \varphi_{l}^{n}=0$, and thus define resonant energies $E^{j}=E_{\mathrm{P}[\mathrm{AP}]}^{\uparrow}=$ $E_{\mathrm{P}[\mathrm{AP}]}^{\downarrow, j}$ for $\gamma_{\mathrm{H}}=0$. From equation (3), the resonant peaks in the conductance curves are spaced by $\Delta E=E^{j+1}-E^{j}=$ $h v_{\mathrm{Fw}} / 2 \ell$ which is usually called the intrinsic level spacing of the wire. Figure 4 shows with black dashed lines the conductance $G^{\mathrm{P}}\left(V_{\mathrm{g}}\right)$ and the magnetoresistance $\operatorname{MR}\left(V_{\mathrm{g}}\right)$ of a one-channel wire. For convenience, we have plotted the physical quantities as a function of $\delta_{0}$ instead of the gate voltage $V_{\mathrm{g}}$. The conductance shows resonances with a $\pi$ periodicity in $\delta_{0}$, corresponding to the intrinsic level spacing $\Delta E$. Strikingly, the magnetoresistance can become negative for certain values of $V_{\mathrm{g}}$ which correspond to a resonance in $G^{\mathrm{P}}$. This is in contrast with the early Jullière's model evoked in section 2. In order to understand this situation (see figure 3), 

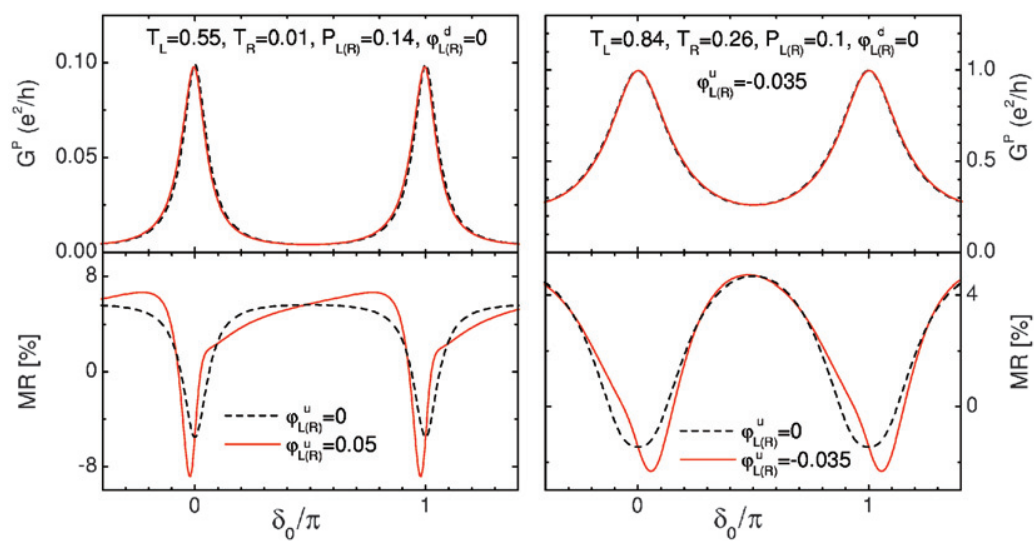

Figure 4. Linear conductance $G^{\mathrm{P}}$ (top panels) and magnetoresistance MR (bottom panels) for a single-channel wire as a function of the spin-averaged phase $\delta_{0}$ acquired by electrons upon crossing the wire ( $\delta_{0}$ is linear with $V_{\mathrm{g}}$ in the limit considered here, see the text). We show the results in the case of no SDIPS (black (dashed) lines) and for a value of SDIPS finite but not resolvable in the conductance curves (red (full) lines). The left and right panels correspond to two different sets of parameters. When the contacts have no SDIPS, the oscillations in $\operatorname{MR}\left(\delta_{0}\right)$ are symmetric. A low SDIPS $\left(\left|\Delta \varphi^{\mathrm{P}[\mathrm{AP}]}\right| \lesssim T_{\mathrm{L}}+T_{\mathrm{R}}\right)$ can be detected qualitatively in the $\operatorname{MR}\left(\delta_{0}\right)$ curves because it breaks the symmetry of these oscillations. In sections 5.2.1 and 5.2.3, we will compare these theoretical results with measurements done in SWNTs connected to PdNi contacts.

it is convenient to consider the limit of low transmissions $T_{l} \ll 1$, in which one can expand $\mathbb{T}_{\mathrm{P}[\mathrm{AP}]}^{\sigma}$ around $E=E^{j}$ (see [9]) to obtain a Breit-Wigner-like formula [12]

$$
\mathbb{T}_{\mathrm{P}[\mathrm{AP}]}^{\sigma}=\frac{T_{\mathrm{L}}^{\sigma} T_{\mathrm{R}}^{\sigma}}{\left(\pi N_{\mathrm{Fw}}\left[E-E^{j}\right]\right)^{2}+\left(T_{\mathrm{L}}^{\sigma}+T_{\mathrm{R}}^{\sigma}\right)^{2} / 4} .
$$

Off resonance, i.e. when $\left(E-E^{j}\right)^{2} \gg\left(T_{\mathrm{L}}^{\sigma}+T_{\mathrm{R}}^{\sigma}\right) / \pi N_{\mathrm{FW}}$, the transmission probability $\mathbb{T}_{\mathrm{P}[\mathrm{AP}]}^{\sigma}$ of the contact for electrons with spin $\sigma$ is proportional to $T_{\mathrm{L}}^{\sigma} T_{\mathrm{R}}^{\sigma}$. This leads to

$$
\mathrm{MR}=\frac{2 P_{\mathrm{L}} P_{\mathrm{R}}}{1-P_{\mathrm{L}} P_{\mathrm{R}}}
$$

as in Jullière's model. At resonance, i.e. when $E=E^{j}$, the situation is different. We will consider for simplicity the very asymmetric case $T_{\mathrm{L}}^{\sigma} \ll T_{\mathrm{R}}^{\sigma}$. In this case, equation (4) gives $\mathbb{T}_{\mathrm{P}[\mathrm{AP}]}^{\sigma}=4 T_{\mathrm{L}}^{\sigma} / T_{\mathrm{R}}^{\sigma}$, which leads to

$$
\mathrm{MR}=-\frac{2 P_{\mathrm{L}} P_{\mathrm{R}}}{1+P_{\mathrm{L}} P_{\mathrm{R}}}
$$

Thus, it clearly appears that the change of sign in the MR signal is a direct consequence of the existence of quasi-bound states in the wire.

Role of the spin dependence of interfacial phase shifts (SDIPS). So far, we have assumed that the interfacial reflection phases of electrons of channel $n$ coming from the wire were spin independent, i.e. $\varphi_{l}^{\uparrow}=\varphi_{l}^{\downarrow}$ for $l \in\{\mathrm{L}, \mathrm{R}\}$. Nevertheless, the interface between a ferromagnet and a nonmagnetic material can scatter electrons with spin parallel or antiparallel to the magnetization of the ferromagnet with different phase shifts, because electrons are affected by a spin-dependent scattering potential at this interface. This spin dependence of interfacial phase shifts can modify significantly the behaviour of many different types of mesoscopic circuits, such as diffusive ferromagnetic/normal/ferromagnetic spin valves [13], superconducting/ferromagnetic hybrid circuits [14], Coulomb blockade systems [15, 16] and Luttinger liquids [17]. Reference [18] has shown that non-interacting ballistic wires can also be affected by the SDIPS. From equation (3), in the case of a finite SDIPS, the resonant energies of the onechannel wire are spin dependent. This allows us to define an SDIPS-induced effective field $h_{\text {SDIPS }}^{c}$ such that

$$
E_{c}^{\downarrow, j}-E_{c}^{\uparrow, j}=g \mu_{\mathrm{B}} h_{\mathrm{SDIPS}}^{c}=\frac{\eta_{\mathrm{L}} \Delta \varphi_{\mathrm{L}}+\eta_{\mathrm{R}} \Delta \varphi_{\mathrm{R}}}{\pi N_{\mathrm{FW}}}
$$

for $c \in\{\mathrm{P}, \mathrm{AP}\}$. Importantly, this effective field is configuration dependent. For instance, in the case of symmetric barriers, one has $\Delta \varphi_{\mathrm{L}}=\Delta \varphi_{\mathrm{R}}$, thus $h_{\mathrm{SDIPS}}^{\mathrm{P}}$ can be finite in the parallel case, but $h_{\mathrm{SDIPS}}^{\mathrm{AP}}$ must vanish in the antiparallel case due to the symmetry of the problem.

The effects of the effective field $h_{\text {SDIPS }}^{c}$ will depend on its amplitude. Let us first imagine that the SDIPS effective field is very strong in the parallel case, i.e. $\left|\Delta \varphi_{\mathrm{L}}+\Delta \varphi_{\mathrm{R}}\right| \gtrsim T_{\mathrm{L}}+T_{\mathrm{R}}$, and that the barriers are symmetric, leading to $h_{\mathrm{SDIPS}}^{\mathrm{AP}}=0$ (one can imagine obtaining this situation by fabricating a symmetric device with strongly spin-dependent barriers, by using e.g. ferromagnetic insulators evaporated between the wire and the metallic contacts). From equation (2), this would allow us to resolve the level spin splitting $g \mu_{\mathrm{B}} h_{\text {SDIPS }}^{\mathrm{P}}$ in the conductance curve $G^{\mathrm{P}}\left(V_{\mathrm{g}}\right)$, and thus to obtain a shift of the conductance peaks from the $\mathrm{P}$ to the AP configurations. A giant MR effect with a sign tunable with $V_{\mathrm{g}}$ would thus be obtained. This illustrates that a strong SDIPS would be very useful for realizing efficient control of spin-dependent transport in nanowires. Nevertheless, since the appropriate device fabrication has not been achieved yet, we refer the reader to [18] for a detailed study of the effects of a strong SDIPS and focus here on the case of a weaker SDIPS. It is possible that a weak SDIPS occurs in actual experiments. Indeed, when a standard ferromagnetic contact material is evaporated directly on a wire, the interfacial scattering potential which affects the electrons can already depend on spin.

Figure 4, red (full) lines, shows the conductance $G^{\mathrm{P}}\left(V_{\mathrm{g}}\right)$ (top panels) and the magnetoresistance $\operatorname{MR}\left(V_{\mathrm{g}}\right)$ (bottom panels) for a device with a weak SDIPS. Although the SDIPSinduced spin splitting is too weak to be resolved in the conductance curves for the parameters used here, it modifies qualitatively the spin-valve behaviour of the device. Indeed, 


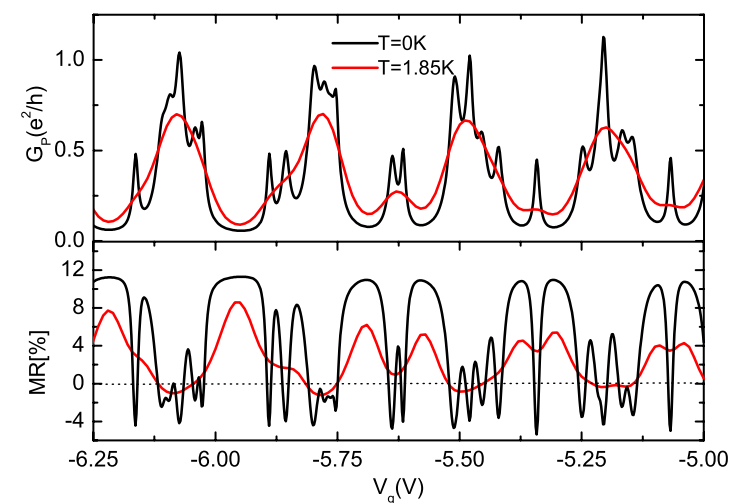

Figure 5. Conductance $G^{\mathrm{P}}$ (top panel) and magnetoresistance MR (bottom panel) calculated for a MWNT with two ferromagnetic contacts, for $T=0$ (black lines) and $T=1.85 \mathrm{~K}$ (red (grey) lines). We have assumed that current transport occurs through six independent channels. We have used $P_{\mathrm{L}(\mathrm{R})}=0.2, T_{\mathrm{L}}=0.45, T_{\mathrm{R}}=$ 0.04 and $\alpha=0.01$ for all channels. A radius of $R_{\mathrm{nt}}=2.7 \mathrm{~nm}$ and a Fermi level of $E_{\mathrm{w}}^{\mathrm{F}}=1 \mathrm{eV}$ have been used for the nanotube in order to calculate the wave vector $k_{\mathrm{FW}}^{n}$ associated with channel $n \in$ $\{1, \ldots, 6\}$. Beatings occur in the zero-temperature signals, due to the combination of the different channels. Therefore, at $T=1.85$ $\mathrm{K}$, the MR signal seems to oscillate with a period which is much larger than the intrinsic spacing between the resonant levels.

when there is no SDIPS, from equation (2), the $\operatorname{MR}\left(V_{\mathrm{g}}\right)$ oscillations are always symmetric with $V_{\mathrm{g}}$. This symmetry is broken by the SDIPS. This is due to the fact that, in the presence of a weak SDIPS, the position of the global maximum corresponding to $E_{c}^{\uparrow, j}$ and $E_{c}^{\downarrow, j}$ is different for $c=\mathrm{P}$ and $c=$ AP. This effect provides a qualitative way to detect the presence of a finite SDIPS in the circuit.

3.1.3. Multichannel case. For MWNTs, it is usually assumed that transport occurs mainly through the outer shell [19]. However, since the diameter of MWNTs is larger than that of SWNTs, the spacing between the 1D subbands of the outer shell is lower [20]. As a consequence, a multichannel description is a priori needed if one wants to account for MR. We have evaluated the conductance and MR from equations (1) and (2), for a MWNT with two ferromagnetic contacts. In the simple case where there is no subband mixing, one can determine the transmission $\mathbb{T}_{\mathrm{P}[\mathrm{AP}]}^{n, \sigma}(E)$ occurring in these equations via the wave vector $k_{\mathrm{FW}}^{n}\left(V_{\mathrm{g}}\right)=k_{\mathrm{Fw}}+\sqrt{\left(e \alpha V_{\mathrm{g}}-E_{\mathrm{FW}}\right)^{2} /\left(\hbar v_{\mathrm{FW}}\right)^{2}-n^{2} / R_{\mathrm{nt}}^{2}}$, where $R_{\mathrm{nt}}$ is the radius of the MWNT [21]. For a radius $R_{\mathrm{nt}}=2.7 \mathrm{~nm}$, the subband spacing amounts to $\sim 180 \mathrm{meV}$. As the Fermi energy shift of MWNTs due to surface adsorbates can be as high as $\sim 1 \mathrm{eV}$ [22], up to six subbands can contribute to charge and spin transport. We have thus taken into account six subbands in the calculation. Figure 5 shows the calculated conductance $G^{\mathrm{P}}$ (top panel) and the magnetoresistance MR (bottom panel) for $T=0$ (black lines) and $T=1.85 \mathrm{~K}$ (red (grey) lines). We have used contact parameters $P_{\mathrm{L}(\mathrm{R})}=$ $0.2, T_{\mathrm{L}}=0.45$ and $T_{\mathrm{R}}=0.04$ for all the channels and $\mathrm{a}$ coupling $\alpha=0.01$. For simplicity, we have assumed no SDIPS. At $T=0$, the conductance shows peaks which correspond to the resonant states in the different channels. Due to the larger number of channels, the intrinsic energy spacing between these resonances is reduced. As a result of the combination of the different conducting channels, beatings occur for certain regions of gate voltage. Importantly, MR can become negative, for the same reason as in the onechannel case. At $T=1.85 \mathrm{~K}$, it is no longer possible to resolve the single-level resonances. Due to thermal averaging, the conductance and magnetoresistance are roughly determined by the envelope of the transmissions. Therefore, the magnetoresistance shows approximately periodic sign changes with a period which is much larger than the intrinsic energy spacing between the resonant states. This type of behaviour will be illustrated with measurements performed with MWNTs in section 5.2.2.

\subsection{Spin-dependent transport in a quantum dot}

The tunnel junctions connecting the ferromagnetic leads to the nanotubes often have a small capacitance of the order of $10 \mathrm{aF}$. In such a case, at low temperatures $(T<10 \mathrm{~K})$, a finite charging energy $U=e^{2} / C_{\Sigma}$ is required to add electrons on a nanotube. The interplay of the Coulomb blockade and spin-dependent transport phenomena has attracted a lot of theoretical and experimental interest recently (see for instance $[15,23-28])$. We introduce below a very recent theoretical development [16] which allows us to address spin transport in the Coulomb blockade regime corresponding to experiment [29].

We assume that strong Coulomb interactions are added to the wire of figure 2 , so that we have a quantum dot connected to ferromagnetic leads. In the interacting regime, the scattering approach used in the former section is no longer suitable for describing this system. One can adopt a description based on the Anderson-like Hamiltonian

$$
H=H_{\text {dot }}+H_{\text {leads }}+H_{\mathrm{c}},
$$

with

$$
\begin{gathered}
H_{\mathrm{dot}}=\sum_{d, \sigma} \xi_{d \sigma} c_{d \sigma}^{\dagger} c_{d \sigma}+\sum_{d, d^{\prime}, \sigma, \sigma^{\prime}\left[(d, \sigma) \neq\left(d^{\prime}, \sigma^{\prime}\right)\right]} \frac{U}{2} n_{d \sigma} n_{d^{\prime} \sigma^{\prime}}, \\
H_{\text {leads }}=\sum_{k, \sigma} \xi_{k \sigma} c_{k \sigma}^{\dagger} c_{k \sigma}, \\
H_{\mathrm{c}}=\sum_{d, k, \sigma}\left(t_{d \sigma}^{k} c_{d \sigma}^{\dagger} c_{k \sigma}+\left(t_{d \sigma}^{k}\right)^{*} c_{k \sigma}^{\dagger} c_{d \sigma}\right) .
\end{gathered}
$$

Here, $\xi_{d \sigma}$ refers to the energy of the dot orbital state $d$ for spin $\sigma, \xi_{k \sigma}$ to the energy of lead state $k$ for spin $\sigma$ and $t_{d \sigma}^{k}$ is a hopping matrix element. The index $k$ runs over the electronic states of leads $\mathrm{L}$ and $\mathrm{R}$. We assume that the spin $\sigma$ is preserved upon tunnelling, as in section 3.1. Coulomb interactions are taken into account through the term in $U$, with $n_{d \sigma}=c_{d \sigma}^{\dagger} c_{d \sigma}$.

Although the notion of interfacial scattering phase is less natural here than with the scattering approach of section 3.1, it is possible to take into account the effects related to the SDIPS in the present interacting model. Indeed, by construction of Hamiltonian (5), for $U=0$, each orbital level $\xi_{d \sigma}$ corresponds to a resonant level $E_{c}^{\sigma, j}$ of section 3.1, with $\xi_{d \downarrow}-\xi_{d \uparrow}=$ $g \mu_{\mathrm{B}} h_{\mathrm{SDIPS}}^{c}$. One can therefore introduce the effective Zeeman splitting $h_{\text {SDIPS }}^{c}$ in equation (5) as a generalization of the SDIPS concept to the interacting case. This can be justified physically on the following basis. In the non-interacting case, we have considered that the ferromagnetic exchange field leads 
to a spin-dependent interfacial potential, responsible for the spin-dependent scattering. For a double barrier system, the ferromagnetic exchange field makes the confinement potential of electrons on the dot spin dependent as well. This naturally induces a spin dependence of the orbital energies, which is the counterpart of the spin splitting of the resonant energies found in section 3.1.

In the interacting case, the zero-bias conductance of the circuit can be expressed as [30]

$$
\begin{aligned}
\frac{h}{e^{2}} \frac{G^{c}}{2} & =\sum_{d, \sigma} \int_{-\infty}^{+\infty} \mathrm{d} \omega \frac{\partial f(\hbar \omega)}{\partial \omega} \\
& \times \frac{\Gamma_{d \sigma}^{\mathrm{L}}(\hbar \omega) \Gamma_{d \sigma}^{\mathrm{R}}(\hbar \omega)}{\Gamma_{d \sigma}^{\mathrm{L}}(\hbar \omega)+\Gamma_{d \sigma}^{\mathrm{R}}(\hbar \omega)} \operatorname{Im}\left[G_{d \sigma}(\omega)\right]
\end{aligned}
$$

with, for $l \in\{\mathrm{L}, \mathrm{R}\}, \Gamma_{d \sigma}^{1}(\xi)=\sum_{k} 2 \pi\left|t_{d \sigma}^{k}\right|^{2} \delta\left(\xi=\xi_{k \sigma}\right)$, and $G_{d \sigma}(\omega)=\int_{-\infty}^{+\infty} G_{d \sigma}(t) \mathrm{e}^{\mathrm{i} \omega t} \mathrm{~d} t$ with $G_{d \sigma}(t)=-\mathrm{i} \theta(t)\left\langle\left\{c_{d \sigma}(t)\right.\right.$, $\left.\left.c_{d \sigma}^{\dagger}(0)\right\}\right\rangle$. For comparison with the experimental data of [29], one has to study current transport in the limit in which the width of conductance peaks displayed by the circuit is not limited only by temperature but also by the tunnel rates $\left(k_{\mathrm{B}} T<\Gamma_{d \sigma}^{\mathrm{L}}+\Gamma_{d \sigma}^{\mathrm{R}}\right)$. This requires going beyond the sequential tunnelling description (see for instance [28]), i.e. also taking into account high-order quantum tunnelling processes. For temperatures larger than the Kondo temperature of the circuit ( $T>T_{\mathrm{K}}$ ), this can be done by calculating Green's functions $G_{d \sigma}(\omega)$ with the equation of motion technique (EOM) introduced for quantum dot systems by Meir et al [31].

Single-orbital case. For simplicity, we first consider a oneorbital quantum dot. Then, the EOM technique leads to [31]

$$
\begin{gathered}
G_{d \sigma}(\omega)=\frac{\hbar\left(1-\left\langle n_{d \bar{\sigma}}\right\rangle\right)}{\hbar \omega-\xi_{d \sigma}-\Sigma_{d \sigma}^{0}+\frac{U \Sigma_{d \bar{\sigma}}^{1}}{\hbar \omega-\xi_{d \sigma}-U-\Sigma_{d \sigma}^{0}-\Sigma_{d \bar{\sigma}}^{3}}} \\
+\frac{\hbar\left\langle n_{d \bar{\sigma}}\right\rangle}{\hbar \omega-\xi_{d \sigma}-U-\Sigma_{d \sigma}^{0}-\frac{U \Sigma_{d \bar{\sigma}}^{2}}{\hbar \omega-\xi_{d \sigma}-\Sigma_{\sigma}^{0}-\Sigma_{d \bar{\sigma}}^{3}}}
\end{gathered}
$$

where $\left\langle n_{d \sigma}\right\rangle=-\int_{-\infty}^{+\infty} \mathrm{d} \omega f(\hbar \omega) \operatorname{Im}\left[G_{\sigma}^{d}(\omega)\right] / \pi$ is the average occupation of orbital $d$ by electrons with spin $\sigma$. Assuming that the coupling to the leads is energy independent (broadband approximation), one has $\Sigma_{d \sigma}^{0}=$ $-\mathrm{i}\left(\Gamma_{d \sigma}^{\mathrm{L}}+\Gamma_{d \sigma}^{\mathrm{R}}\right) / 2, \Sigma_{d \bar{\sigma}}^{3}=-\mathrm{i}\left(\Gamma_{d \bar{\sigma}}^{\mathrm{L}}+\Gamma_{d \bar{\sigma}}^{\mathrm{R}}\right)$ and, for $i \in\{1,2\}$,

$$
\begin{aligned}
\Sigma_{d \bar{\sigma}}^{i} & =\sum_{k} \frac{\mu_{i}\left(\xi_{k \bar{\sigma}}\right)\left|t_{k \bar{\sigma}}^{d}\right|^{2}}{\hbar \omega-\xi_{d \sigma}+\xi_{d \bar{\sigma}}-\xi_{k \bar{\sigma}}+\mathrm{i} 0^{+}} \\
& +\sum_{k} \frac{\mu_{i}\left(\xi_{k \bar{\sigma}}\right)\left|t_{k \bar{\sigma}}^{d}\right|^{2}}{\hbar \omega-\xi_{d \sigma}-\xi_{d \bar{\sigma}}-n U+\xi_{k \bar{\sigma}}+\mathrm{i} 0^{+}},
\end{aligned}
$$

with $\mu_{1}(\xi)=f(\xi)$ and $\mu_{2}(\xi)=1-f(\xi)$. The term $\Sigma_{d \sigma}^{0}$, which is due to the tunnelling of electrons with spin $\sigma$, already occurred in the non-interacting case. Indeed, for $U=0$ and $T_{l}^{\sigma} \ll 1$, the conductance given by the above equations can be perfectly mapped onto the non-interacting conductance found in section 3.1, using $E_{d \sigma}^{c}=\xi_{d \sigma}$ and $T_{l}^{\sigma}=\pi N_{\mathrm{FW}} \Gamma_{d \sigma}^{l}=2 \pi N_{\mathrm{FW}}\left|\Sigma_{d \sigma}^{0}\right|$. In the interacting case, $G_{d \sigma}(\omega)$ also involves $\Sigma_{d \sigma, d^{\prime} \sigma^{\prime}}^{i, n}$ terms related to the tunnelling of electrons with spin $\bar{\sigma}$. Note that $G_{d \sigma}, \xi_{d \sigma}$ and $\Gamma_{d \sigma}^{L(R)}$ depend on the configuration $c \in\{\mathrm{P}, \mathrm{AP}\}$ considered, but for simplicity we have omitted the index $c$ in those quantities.

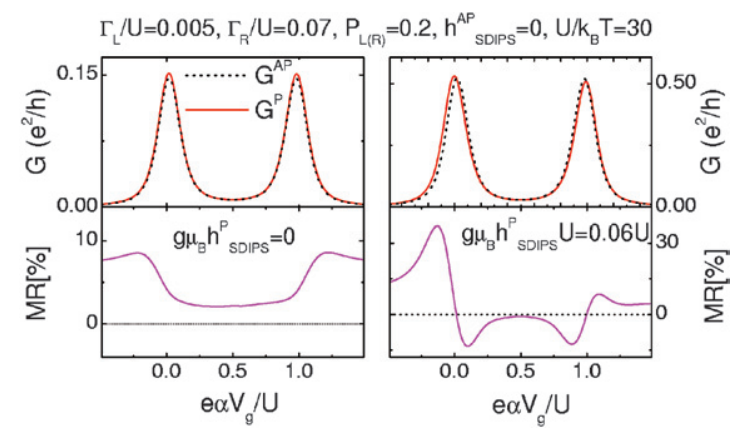

Figure 6. Top panels: conductance $G^{\mathrm{P}}$ in the parallel configuration (red (full) lines) and conductance $G^{\mathrm{AP}}$ in the antiparallel configuration (black (dotted) lines) as a function of the gate voltage $V_{\mathrm{g}}$, for the circuit shown in figure 2 , with w a one-orbital quantum dot. We have used $\Gamma_{\mathrm{L}}=0.005 U, \Gamma_{\mathrm{R}}=0.07 U, P_{\mathrm{L}(\mathrm{R})}=0.2$, $U / k_{\mathrm{B}} T=30$ and $h_{\mathrm{SDIPS}}^{\mathrm{AP}}=0$. Bottom panels: magnetoresistance MR (pink (grey) curves) corresponding to the above conductance plots. The results are shown for $g \mu_{\mathrm{B}} h_{\mathrm{SDIPS}}^{\mathrm{P}}=0$ (left panels) and $g \mu_{\mathrm{B}} h_{\mathrm{SDIPS}}^{\mathrm{P}}=0.06 U$ (right panels).

Figure 6 shows the conductance $G^{c}$ in configuration $c \in\{\mathrm{P}, \mathrm{AP}\}$ (top panels) and the magnetoresistance MR (bottom panels) calculated for different values of $h_{\text {SDIPS }}^{c}$, using $\Gamma_{d \sigma}^{l}=\Gamma_{l}\left(1+\eta_{l} \sigma P_{l}\right)$ for $l \in\{\mathrm{L}, \mathrm{R}\}$. The conductance peak corresponding to level $d$ is split by $U$ due to Coulomb interactions. For $h_{\text {SDIPS }}^{c}=0$ (left panels), we already note that although the two conductance peaks displayed by $G^{\mathrm{P}}\left(V_{\mathrm{g}}\right)$ are very similar, the MR variations corresponding to these two peaks have different shapes (see also [24]). More precisely, for the low values of polarization considered here, $\operatorname{MR}\left(V_{\mathrm{g}}\right)$ is approximately mirror symmetric from one conductance peak to the other. This is in contrast with the single-channel non-interacting case in which $\operatorname{MR}\left(V_{\mathrm{g}}\right)$ was identical for all conductance peaks. A finite effective field $h_{\text {SDIPS }}^{c}$ produces a shift of the conductance peaks from the $\mathrm{P}$ to the AP configurations. For instance, in figure 6, top-right panel, the left (right) conductance peak is shifted to the right (left) from $\mathrm{P}$ to AP because it comes in majority from the transport of up (down) spins in the $\mathrm{P}$ case. As a consequence, in figure 6, bottom-right panel, the amplitude of MR is enhanced and it becomes negative for certain values of $V_{\mathrm{g}}$. We note that using a finite SDIPS does not help to obtain similar MR variations for the two peaks of $G^{\mathrm{P}}\left(V_{\mathrm{g}}\right)$ because the SDIPS shifts these two peaks in opposite directions.

Before concluding this section, we point out that, in principle, $h_{\text {SDIPS }}^{c}$ is not the only term which can lead to a spin splitting of the dot energy levels. Indeed, the terms proportional to $\Sigma_{d \bar{\sigma}}^{1}$ and $\Sigma_{d \bar{\sigma}}^{2}$ in equation (9) can also renormalize these levels, due to their real part. In the case of ferromagnetic contacts, this renormalization is different for the two spin directions. This allows us to define another type of effective field, $h_{U}^{c}$, which is intrinsically taken into account in the treatment shown here. The effects of $h_{U}^{c}$ have been studied in detail by [25] for a quantum dot with noncollinearly polarized ferromagnetic leads in the sequential tunnelling regime (see reference [34] of [16]) and by [27] for a quantum dot in the Kondo regime. Similarly to $h_{\text {SDIPS }}^{c}$, the value of $h_{U}^{c}$ depends on the configuration of the ferromagnetic electrodes and it must vanish in the AP configuration for symmetric junctions. Nevertheless, for the low values of 


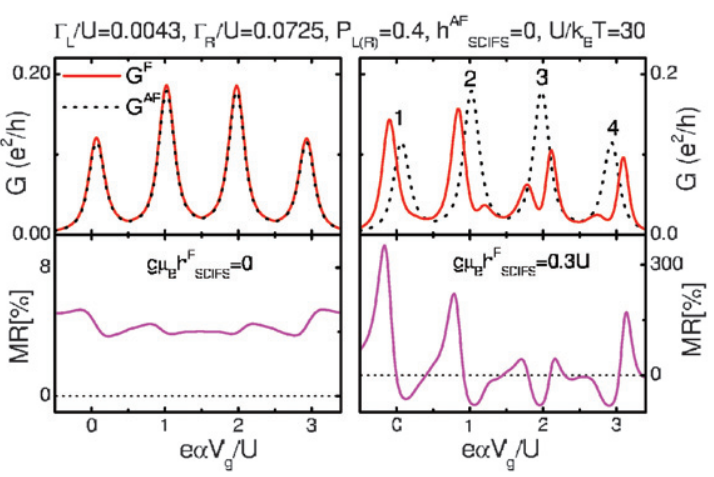

Figure 7. Top panels: conductance $G^{\mathrm{P}}$ in the parallel configuration (red (full) lines) and conductance $G^{\mathrm{AP}}$ in the antiparallel configuration (black (dotted) lines), for the circuit of figure 2, with w a two-orbital quantum dot. We have used identical tunnel rates to the two orbitals, i.e. $\Gamma_{\mathrm{L}}=0.0043 U, \Gamma_{\mathrm{R}}=0.0725 U$ and

$P_{\mathrm{L}(\mathrm{R})}=0.4$. We have also used $U / k_{\mathrm{B}} T=30$ and $h_{\mathrm{SDIPS}}^{\mathrm{AP}}=0$. Bottom panels: magnetoresistance MR (pink (full) lines) corresponding to the conductance plots. The results are shown for $g \mu_{\mathrm{B}} h_{\mathrm{SDIPS}}^{\mathrm{P}}=0$ (left panels) and $g \mu_{\mathrm{B}} h_{\mathrm{SDIPS}}^{\mathrm{P}}=0.3 U$ (right panels). The two-fold orbital degeneracy allows the restoration locally of an approximate regularity of the $\operatorname{MR}\left(V_{\mathrm{g}}\right)$ pattern. This behaviour will be compared with experimental results in section 5.2.3.

tunnel rates $\Gamma_{\mathrm{L}(\mathrm{R})}$, polarizations $P_{\mathrm{L}(\mathrm{R})}$ and temperatures $T$ used here, $h_{U}^{c}$ is much weaker than the finite $h_{\text {SDIPS }}^{c}$ assumed, and it can therefore not play the same role as $h_{\text {SDIPS }}^{c}$.

Generalization to a non-degenerate multilevel system. For simplicity, we have considered in the previous section the oneorbital case. In practice, other orbital levels close to orbital $d$ can modify the $\operatorname{MR}\left(V_{\mathrm{g}}\right)$ pattern. Nevertheless, for nondegenerate energy levels with a sufficiently large intrinsic level spacing $\Delta E$ (see [16]), the two conductance peaks associated with a given orbital will occur consecutively in $G^{c}\left(V_{\mathrm{g}}\right)$. The SDIPS will shift these two peaks in the same way as for the single-orbital model. Therefore, one can still expect changes of sign in the $\operatorname{MR}\left(V_{\mathrm{g}}\right)$ curves, with dissimilar $\operatorname{MR}\left(V_{\mathrm{g}}\right)$ patterns for the two conductance peaks corresponding to a given orbital level.

Effect of a two-fold degeneracy of orbital levels. In single-wall carbon nanotubes, a two-fold orbital degeneracy is commonly observed, related to the $K-K^{\prime}$ energy band degeneracy of graphene $[10,11]$. To investigate some consequences of this feature, one can consider a two-degenerate-orbitals model, i.e. Hamiltonian (5) with $d \in\left\{K, K^{\prime}\right\}$ and $\xi_{K^{\prime} \sigma}=\xi_{K \sigma}$. For simplicity, we assume no coupling between the two orbitals through higher order dot-lead tunnel processes. We also assume the same dot-lead coupling and interfacial parameters for both orbitals. In the non-interacting case, this modification leaves the MR unchanged (see section 3.1). In the interacting limit, an orbital degeneracy has more complicated effects on MR. This was studied with the EOM technique in [16]. We refer the readers to this reference for details of the calculation and present here the main results of this approach.

Figure 7 shows the conductance (top panels) and MR curves (bottom panels) in the two-orbital case, calculated for

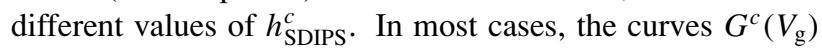
show four resonances, the first two associated with a single occupation of $K$ and $K^{\prime}$, and the other two with double occupation. For $h_{\text {SDIPS }}^{\mathrm{P}}=h_{\mathrm{SDIPS}}^{\mathrm{AP}}=0$ and the parameters used here, MR remains positive for any value of $V_{\mathrm{g}}$ (left panels). As in the one-orbital case, a finite $h_{\text {SDIPS }}^{c}$ makes easier negative MR effects and allows a stronger tunability of the MR effect with $V_{\mathrm{g}}$ (right panels). Importantly, the effect of $h_{\text {SDIPS }}^{c}$ again depends on the occupation of the dot and the $\operatorname{MR}\left(V_{\mathrm{g}}\right)$ pattern is thus not similar for the four conductance peaks. Nevertheless, in figure 7, top-right panel, the first two conductance peaks of $G^{\mathrm{P}}$ (peaks 1 and 2) are both shifted to the left by $h_{\text {SDIPS }}^{\mathrm{P}}$ because they are both due in majority to up spins. This allows us to get a MR pattern approximately similar for these two peaks, i.e. a transition from positive to negative values of MR (bottom-right panel). In contrast, peaks 3 and 4 correspond to a transition from negative to positive values of MR because the associated conductance peaks are due in majority to down spins. The shape of the $\operatorname{MR}\left(V_{\mathrm{g}}\right)$ pattern associated with the transition between peaks 3 and 4 is particular (negative/positive/negative) because, for the values of parameters considered here, the Coulomb blockade does not entirely suppress the up spins' contribution in peak 3. Remarkably, this allows us to obtain, at the left of figure 7, bottom-right panel, three positive MR maxima which differ in amplitude but have rather similar shapes. Taking into account a two-fold orbital degeneracy thus allows us to restore an approximate local regularity of the MR pattern. Note that for clarity we have used in figure 7 , right panels, a large value of

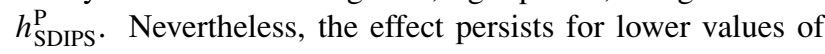
SDIPS (see figure 14). This behaviour will be compared with the experimental data of [29] in section 5.2.3.

\subsection{Other interacting regimes}

In sections 3.1 and 3.2, we have put special emphasis on the non-interacting regime and on the Coulomb blockade regime because this is relevant for interpreting the experimental results available so far (see section 5). However, in principle, a carbon nanotube connected to ferromagnetic leads can adopt other types of behaviour. For instance, it could behave as a quantum dot in the Kondo regime (see e.g. [32]). The effect of ferromagnetic leads on this system has been studied theoretically by various authors [26, 27]. A first experimental study could be realized using $\mathrm{C}_{60}$ molecules, revealing a spin splitting of the Kondo resonance related to the coupling to the ferromagnetic leads [33]. Nevertheless, in carbon nanotubes, the Kondo effect has only been observed with non-magnetic leads so far [34]. A carbon nanotube can also behave as a Luttinger liquid due to the interplay between electronic interactions and the one-dimensional nature of the nanotube $[35,36]$. In a Luttinger liquid, electrons form collective charge and spin excitations which propagate with different velocities. The resulting spin-charge separation effect remains to be observed in an unambiguously accepted way [37]. This is one more fundamental motivation for the study of spin transport in carbon nanotubes. It has been predicted that spin transport could provide experimental evidence of spin-charge separation, in the Fabry-Perot-like regime [38] corresponding to section 3.1, as well as the incoherent regime $\max \left(e V_{\mathrm{sd}}, k_{\mathrm{B}} T\right) \gg h v_{\mathrm{Fw}} / \ell$ (see [17]). We will not report on those predictions in detail here because no experimental realization is available at this time. 


\subsection{The spin injection problem}

Until now, we have assumed that it was possible to inject spins reliably in carbon nanotubes. More precisely, in the framework of the theories presented in sections 3.1 and 3.2, we have assumed that the spin polarization $P_{l}^{n}$ of the tunnel rates was finite. One must wonder whether this is possible in practice.

In the last decade, the realization of spin injection from ferromagnetic metals into semiconductors has triggered many efforts, motivated by the proposal by Datta and Das for a spin transistor based on the electric field control of spinorbit coupling through the Rashba effect $[2,39]$. One major difficulty came from the problem of the so-called conductivity mismatch. As shown by [40] in the diffusive limit, when a semiconductor is in good contact with a ferromagnetic material, the spin polarization of the current injected into the semiconductor is strongly reduced because the conductivity of the semiconductor is much smaller than that of the ferromagnet. Nevertheless, it was shown that spin injection can be enhanced by adding tunnelling contacts at the interfaces between the ferromagnets and the non-magnetic materials $[41,42]$. This turns out to be valid also in the ballistic limit (see for instance [43]), and in particular for carbon nanotubes, as illustrated in the next section.

3.4.1. The ballistic spin injection picture. Tunnel barriers are commonly obtained between ferromagnetic metals and carbon nanotubes. This makes spin injection possible as we are going to show here. Estimating accurately the spin injection parameter $P_{l}^{n}$ for a ferromagnet/nanotube interface is beyond the scope of this paper. We will rather adopt here a scattering approach with a Dirac potential barrier to model the interface. As shown by [43], this simplified approach is enough to capture some essential properties of the interfaces.

We assume a Dirac-function potential $U_{l}^{\sigma} \delta\left(x-x_{l}\right)$ for the interface $l \in\{\mathrm{L}, \mathrm{R}\}$ placed at $x_{l}$, and we use a spin-dependent wave vector $k_{l}^{\sigma}$ for ferromagnetic lead $l$. Solving the quantum mechanical problem, one finds for junction $l$ a transmission probability

$$
T_{l}^{n, \sigma}=\frac{4 k_{\mathrm{Fw}} k_{l}^{\sigma}}{\left(k_{\mathrm{Fw}}+k_{l}^{\sigma}\right)^{2}+\left(2 U_{l}^{\sigma} m_{\mathrm{e}} / \hbar^{2}\right)^{2}} .
$$

For completeness, we also give the expression of the wire-wire reflection phase

$$
\varphi_{l}^{n, \sigma}=\arctan \left[\frac{2 U_{l}^{\sigma} m_{\mathrm{e}}}{\hbar^{2}\left(k_{\mathrm{Fw}}-k_{l}^{\sigma}\right)}\right]+\arctan \left[\frac{2 U_{l}^{\sigma} m_{\mathrm{e}}}{\hbar^{2}\left(k_{\mathrm{Fw}}+k_{l}^{\sigma}\right)}\right]
$$

at contact $l$. Figure 8 shows the spin-averaged transmission probability $T_{l}^{n}=\left(T_{l}^{n, \uparrow}+T_{l}^{n, \downarrow}\right) / 2$, the spin polarization of the transmission probability $P_{l}^{n}=\left(T_{l}^{n, \uparrow}-T_{l}^{n, \downarrow}\right) /\left(T_{l}^{n, \uparrow}+T_{l}^{n, \downarrow}\right)$ and the SDIPS parameter $\Delta \varphi_{l}^{n}=\varphi_{l}^{n, \uparrow}-\varphi_{l}^{n, \downarrow}$ calculated from these equations. For the nanotube, we use $k_{\mathrm{Fw}} \sim 8.5 \times 10^{9} \mathrm{~m}^{-1}$ [8], and for the ferromagnetic contact, we use the typical value $k_{l}^{\sigma} \sim 1.7 \times 10^{10} \mathrm{~m}^{-1}[43]$ and a spin polarization $p_{l}=0.3$ for the electronic density of states in lead $l$. We define the average barrier strength $Z_{l}=m_{\mathrm{e}}\left(U_{l}^{\uparrow}+U_{l}^{\downarrow}\right) / \hbar^{2} k_{\mathrm{Fw}}$. We first assume that $U_{l}^{\sigma}$ is spin independent (full curves). For a metallic contact, that is $Z_{l}=0, P_{l}^{n}$ remains very small. Nevertheless, the spin injection efficiency is strongly enhanced for a high
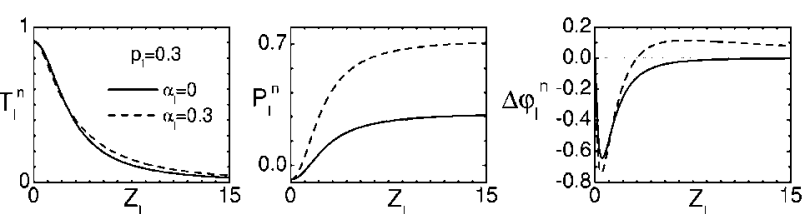

Figure 8. Spin-averaged tunnelling rate $T_{l}^{n}$ (left panel), tunnelling rate polarization $P_{l}^{n}$ (middle panel) and SDIPS parameter $\Delta \varphi_{l}^{n}$ (right panel) of contact $l \in\{\mathrm{L}, \mathrm{R}\}$, estimated by using a Dirac barrier model with a spin-dependent coefficient $U_{l}^{\sigma}$, placed between a ferromagnetic metal with Fermi wave vector $k_{l}^{\sigma} \sim 1.7 \times 10^{10} \mathrm{~m}^{-1}$ and a wire with Fermi wave vector $k_{\mathrm{Fw}}=8.5 \times 10^{9} \mathrm{~m}^{-1}$ typical of single-wall nanotubes. We show the results as a function of the average barrier strength $Z_{l}=m_{\mathrm{e}}\left(U_{l}^{\uparrow}+U_{l}^{\downarrow}\right) / \hbar^{2} k_{\mathrm{Fw}}$, for a polarization $p_{l}=0.3$ of the lead density of states and different values of the spin asymmetry $\alpha_{l}=\left(U_{l}^{\downarrow}-U_{l}^{\uparrow}\right) /\left(U_{l}^{\uparrow}+U_{l}^{\downarrow}\right)$ of the barrier.

barrier strength [43]. It is also possible that the potential barrier between the nanotube and the ferromagnet is itself spin polarized, i.e. $\alpha_{l}=\left(U_{l}^{\downarrow}-U_{l}^{\uparrow}\right) /\left(U_{l}^{\uparrow}+U_{l}^{\downarrow}\right) \neq 0$. This can be due to the magnetic properties of the contact material itself, when it is evaporated directly on the nanotube, but it can also be obtained artificially by using a magnetic insulator (see [44]) to form the barrier. This allows us to further enhance spin injection (dashed lines).

Before concluding this section, we point out that in the case of coherent quantum transport, $P_{l}^{n}$ is not the only parameter which sets the efficiency of spin injection. Indeed, $\Delta \varphi_{l}^{n}$ is also a crucial parameter since it determines the localized quantum states inside the wire. Let us consider for simplicity the non-interacting case of section 3.1. For the weak values of SDIPS used in figure 4, spin injection was not improved. However, it was shown that with a stronger SDIPS, the resonant states in $G^{\mathrm{P}[\mathrm{AP}]}$ are spin split, each subpeak corresponding to a given spin direction (see [18]). Having a strong SDIPS can thus allow us to have a strongly spin-polarized current. Since the SDIPS-induced spin splitting is different in the $\mathrm{P}$ and AP configurations, this allows us to further increase the MR. One can see from figure 8 that the condition required for this effect can be obtained with weakly transparent and spin-dependent barriers (see dashed lines for large $Z_{l}$ ), which is compatible with having a large $P_{l}^{n}$.

3.4.2. Experimental identification of spin injection. In sections 3.1 and 3.2, we have considered the gate-tunable MR effect produced by spin injection into the nanotube, i.e. $P_{\mathrm{L}(\mathrm{R})}^{n} \neq 0$. We will introduce in section 4.2 other types of MR effects which are not due to spin injection but to various properties of the ferromagnetic contacts. To prove that spin injection is actually taking place in an experiment, one must be able to discriminate the spin injection MR effect from contact MR effects.

For this purpose, one key idea is that MR found in sections 3.1 and 3.2 is mainly a two-terminal effect. If only one of the contacts $k \in\{\mathrm{L}, \mathrm{R}\}$ is ferromagnetic, spin injection will still induce a hysteresis in $G$ as a function of the external field $H$, but with only a very small change $\Delta G$ when $H= \pm H_{\mathrm{c} k}$. This slight change will occur because the situations in which $\overrightarrow{p_{k}}$ is parallel or antiparallel to $\vec{H}$ are not totally equivalent according to equation (2). For instance, 
using the same parameters as in figure 4 but with $P_{l}=0$ and $\Delta \varphi_{l}=0$ for one of the two contacts $(l=\mathrm{L}$ or $l=\mathrm{R})$ and using the typical value $g \mu_{\mathrm{B}} H_{\mathrm{c} k} L / \hbar v_{\mathrm{Fw}}=0.01$, one finds a value $|\Delta G| / G<0.8 \%$ much weaker than the maximum MR found for two ferromagnetic contacts in this figure. The situation seems to be different for contact MR effects: as we will see in section 4.2, these effects should already be significant with a single ferromagnetic contact if they are relevant with two ferromagnetic contacts. We will describe in section 4.2 other more specific features which can allow the identification of contact MR effects.

\section{Contacting carbon nanotubes with ferromagnetic contacts}

In this section, we present the state of the art in contacting carbon nanotubes with ferromagnetic materials. We emphasize the characteristics of the contacts such as minimum room temperature two-probe resistance (or transmission) and the maximum MR amplitude. We also discuss contact effects which are not related to spin-dependent transport inside the nanotubes but which could sometimes be superimposed on the MR effects described in the previous section.

\subsection{Contacting carbon nanotubes with ferromagnetic leads}

Contacting carbon nanotubes with metallic electrodes has been an issue since the start of the study of their electronic properties. Most transport measurements have been carried out in a two-probe geometry. In the case of non-magnetic electrodes, the transmission of the contacts determines the relevant regime for charge transport. As we have seen above, connecting ferromagnetic contacts to carbon nanotubes raises additional questions, such as e.g. the efficiency of spin injection. Therefore, the choice of the ferromagnet is crucial not only regarding the coupling of the electrodes to the nanotube but also regarding the reliability of spin injection.

In the case of non-magnetic contacts, a few multi-probe measurements have been carried out $[45,46]$ and provide new insights into quantum transport in nanotubes. Similarly, one can expect multi-probe measurements to provide useful information about spin transport when some of the probes are ferromagnetic [47, 48]. We will however focus on the studies of the two-probe geometries since they are the most advanced carried out so far. Table 1 provides a summary of these works.

The main feature to be observed in a carbon nanotube connected to two ferromagnetic leads is a hysteresis of the resistance versus an applied magnetic field swept in two opposite directions, as shown in figure 1, right. One delicate point is the control of the switching of the magnetization. It turns out that it is non-trivial to accurately control the domain pattern of the ferromagnet near the contact with the nanotubes. Therefore, almost no MR curve looks like the theoretical ones. The hysteresis curves often show complex structures. As we will see, this problem has been partially solved with $\mathrm{Ni}_{1-x} \mathrm{Pd}_{x}$ and $\mathrm{La}_{2 / 3} \mathrm{Sr}_{1 / 3} \mathrm{MnO}_{3}$ (LSMO) contacts.

4.1.1. Co contacts. In the pioneering work by Tsukagoshi and co-workers, Co contacts were used to study spindependent transport in MWNTs [49]. The two-terminal resistance of the devices at room temperature ranged from $8 \mathrm{k} \Omega$ to $250 \mathrm{k} \Omega$ [50]. At $4.2 \mathrm{~K}$, the authors observed a MR which they attributed to spin transport along the relatively short MWNT length $(300 \mathrm{~nm})$. The magnetic field was applied inplane. The electrodes were both in Co and had the same geometry. Given that there was a priori no reason for having two different coercive fields in the two electrodes, the AP configuration was difficult to identify. The amplitude of the hysteresis was at most about $+9 \%$. The observed reduced value was attributed to spin relaxation in the nanotube, yielding a spin relaxation length of $l_{\mathrm{s}}=260 \mathrm{~nm}$. The method was extended later to SWNT devices [50]. A few experiments with multi-probes of Co were carried out for SWNTs $[47,48]$. In that case, the two-probe resistance ranged from about $12 \mathrm{k} \Omega$ to $\mathrm{M} \Omega \mathrm{s}$. The two-terminal MR reported ranged from $2 \%$ to $6 \%$. In [48], shape anisotropy was used to selectively control the switching of the different Co electrodes (typical width about $100 \mathrm{~nm}$ ). Experiments with Co electrodes were also carried out by Zhao and co-workers [51] but a negative MR of $-36 \%$ was observed.

It is important to point out that the above experiments have been realized without a gate voltage supply $V_{\mathrm{g}}$. In the absence of any doping, this would imply that the carbon nanotubes were operated at their charge-neutral point. However, it has been found that carbon nanotubes are extremely sensitive to their chemical environment. The chemical potential $E_{\mathrm{Fw}}$ of a nanotube can be strongly modified by surface adsorbates such as water molecules, gas molecules or ions [22, 52, 53]. In sections 3.1 and 3.2, we have shown that the sign and amplitude of MR strongly depend on $E_{\mathrm{Fw}}$. This implies that the spin signal will depend on details of the nanotube environment. One can even expect that MR differs significantly if one measures the same sample in different cool-downs, as observed in another experiment described in the next section [54]. Therefore, although the different signs and amplitudes of MR found in the $\mathrm{Co} /$ nanotube experiments are compatible with the theoretical expectations, a further experimental investigation of the MR effect requires the use of a gate electrode in order to study the dependence of MR on $V_{\mathrm{g}}$, which is much more significant than the value of MR without a gate voltage supply.

4.1.2. Fe contacts. Fe is another possible choice for making ferromagnetic contacts on nanotubes. There is only one study using Fe on SWNTs carried out by Jensen and co-workers $[54,55]$. In that case, the two-terminal resistances reported at room temperature vary from $80 \mathrm{k} \Omega$ to $1 \mathrm{M} \Omega$. In this study, CVD-grown SWNTs were used. The first contacts were made on the top of catalyst squares and had a typical size of $6 \mu \mathrm{m} \times 8 \mu \mathrm{m}$. The second contact design was two $\mathrm{Fe}$ electrodes with different aspect ratios, typically $10 \mu \mathrm{m} \times$ $300 \mathrm{~nm}$ and $10 \mu \mathrm{m} \times 200 \mathrm{~nm}$ in order to control the switching via shape anisotropy. However, both these contact geometries gave similar magnetization switching for a field applied inplane. The samples were coupled to an electrostatic gate. The sign of MR could be changed from positive to negative with the gate voltage. The observed MR ranged from $-50 \%$ up to $100 \%$. Due to the absence of a detailed study of MR versus $V_{\mathrm{g}}$, a clear conclusion cannot be drawn from this work. 

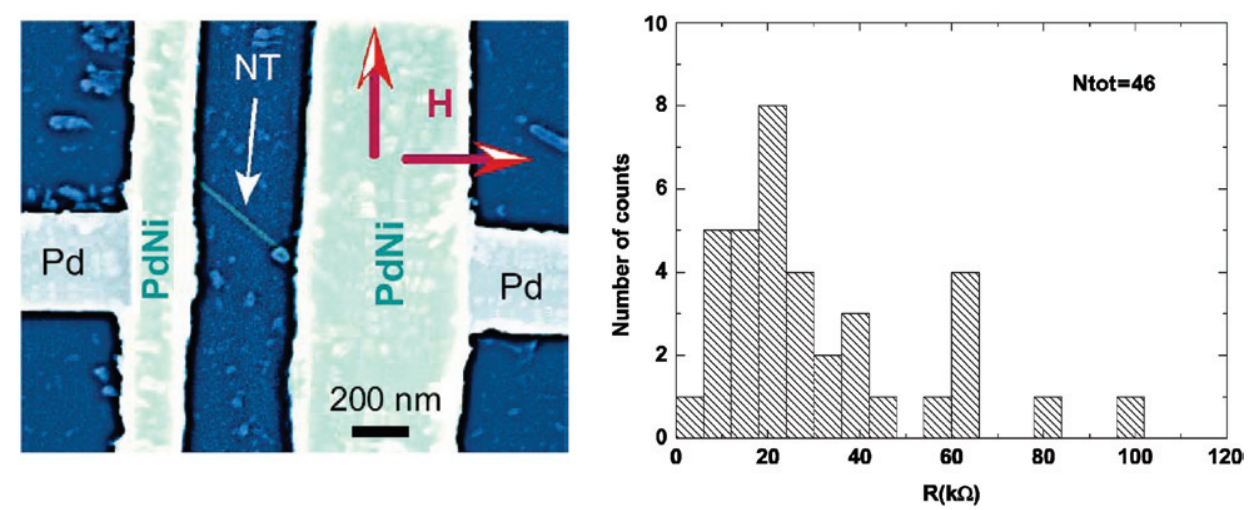

Figure 9. Left: SEM micrograph of a typical F-nanotube-F sample of Sahoo et al [29]. NiPd contacts are used to inject and detect spins electrically in a MWNT with a contact separation of about $400 \mathrm{~nm}$. The external magnetic field is applied in-plane, either perpendicular or parallel to the axis of the elongated NiPd strips. Right: statistics of the contacting properties for NiPd on MWNTs. The typical two-probe resistance at room temperature is $20 \mathrm{k} \Omega$.

Table 1. Summary of the various contacting materials used so far and their contacting properties. Columns 2 and 3 display the minimum two-probe resistances measured at room temperature for SWNTs and MWNTs contacted with the material indicated in column 1. Column 4 displays the maximum MR amplitude measured at low temperatures. Column 5 reports the MR signs observed. Column 6 indicates whether gate control of MR was achieved. Column 7 indicates the magnetic signal $\Delta G / G$ measured for nanotubes contacted with one ferromagnetic lead and one non-magnetic lead. Column 8 indicates the corresponding references $(\mathrm{N}=$ not reported $)$.

\begin{tabular}{lllrllll}
\hline Material & $\begin{array}{l}\text { SWNT } \\
(\mathrm{k} \Omega)\end{array}$ & $\begin{array}{l}\text { MWNT } \\
(\mathrm{k} \Omega)\end{array}$ & $\begin{array}{l}\text { Max }|\mathrm{MR}| \\
(\%)\end{array}$ & MR sign & $\begin{array}{l}\text { Gate } \\
\text { control }\end{array}$ & $\begin{array}{l}\mathrm{F}-\mathrm{NT}-\mathrm{N} \\
\Delta G / G(\%)\end{array}$ & References \\
\hline $\mathrm{Co}$ & 15 & 8 & 36 & + and - & No & $\mathrm{N}$ & {$[47-51]$} \\
$\mathrm{Fe}$ & 80 & $\mathrm{~N}$ & 100 & + and - & Yes & $\sim 0$ & {$[54,55]$} \\
$\mathrm{Ni}$ & $\mathrm{N}$ & $\mathrm{N}$ & 15 & + and - & Yes & $\mathrm{N}$ & {$[56]$} \\
$\mathrm{NiPd}$ & 11 & 5.6 & 17 & + +and - & Yes & $<1.4$ & {$[29,57,58]$} \\
$(\mathrm{Ga}, \mathrm{Mn}) \mathrm{As}$ & $\mathrm{N}$ & $\mathrm{N}$ & 150 & + and - & Yes & $<10$ & {$[54,55]$} \\
LSMO & $\mathrm{N}$ & 1000 & 37 & + & No & $\mathrm{N}$ & {$[60]$} \\
\hline
\end{tabular}

4.1.3. Ni contacts. $\mathrm{Ni}$ has also been used to implement ferromagnetic electrodes on SWNTs [56]. The main finding with respect to the other works is a continuous sign change as a function of gate voltage, from $+10 \%$ to $-15 \%$. Although the channel length was about $10 \mathrm{~nm}$, no size quantization was observed at $4.2 \mathrm{~K}$.

4.1.4. NiPd contacts. In principle, all kinds of ferromagnetic alloys could be tried in order to improve the reliability of the spin injection and/or the switching of the magnetization. Among these choices, Pd-based alloys look particularly promising. Indeed, experiments using $\mathrm{Ni}_{1-x} \mathrm{Pd}_{x}$ with $x \sim 0.5$ are among the most advanced studies for spin transport in carbon nanotubes [29, 57, 58]. This choice is based on the observation that $\mathrm{Pd}$ alone makes reliable contacts on MWNTs as well as SWNTs [59]. Furthermore, Pd is close to the ferromagnetic instability with a Stoner enhancement of about 10. A few magnetic impurities are enough to drive it in the ferromagnetic state (the same holds for $\mathrm{Pt}$ which has a somewhat lower Stoner enhancement of about 4). Therefore, it seems possible to combine the good contacting properties of Pd with a finite spin polarization. Furthermore, the use of $\mathrm{Pd}$ as contacting metal prevents oxide layers from forming at the ferromagnet/nanotube interface. This might be an advantage with respect to the methods using pure ferromagnetic metals, because most of the ferromagnetic oxides are anti-ferromagnetic and therefore not only depolarize the electronic current, but also modify in general the spin activity of the interface.

Sahoo and co-workers [58, 29] were the first to study this contacting scheme on nanotubes. The type of device studied is presented in figure 9. Two ferromagnetic $\mathrm{Pd}_{0.3} \mathrm{Ni}_{0.7}$ strips are used to contact either a MWNT or a SWNT. They have different shapes, typically $14 \mu \mathrm{m} \times 0.1 \mu \mathrm{m}$ and $3 \mu \mathrm{m} \times$ $0.5 \mu \mathrm{m}$ for the left and the right electrode, respectively. The narrower electrode has a sharp switching around 100-250 mT. The wider one has a less pronounced switching, as shown in figure 10. This suggests that its magnetization gradually rotates upon reversing the sign of the external magnetic field. It is worth noting that Man et al [57] as well as Sahoo et al have found that the magnetic anisotropy of the NiPd strips is in-plane, perpendicular to their long axis. This is in contradiction with the expected shape defined anisotropy and might be related to the complexity of the domain structure of the Pd-based ferromagnetic alloys. The two-probe resistance at room temperature of devices with MWNTs studied by Sahoo et al [58] is summarized in the right panel of figure 9 . As shown by this figure, the distribution of resistances is rather peaked at the typical value of $20 \mathrm{k} \Omega$, which shows the reliability of this contacting procedure. The minimum value is $5.6 \mathrm{k} \Omega$, the best ever reported for ferromagnetic contacts on MWNTs. For SWNTs, the transparency of the contacts is lower in general, but transmission probabilities as high as 0.84 have been reported by Man et al [57].

At temperatures below $10 \mathrm{~K}$, a MR is commonly observed upon sweeping an external magnetic field. Depending on the 

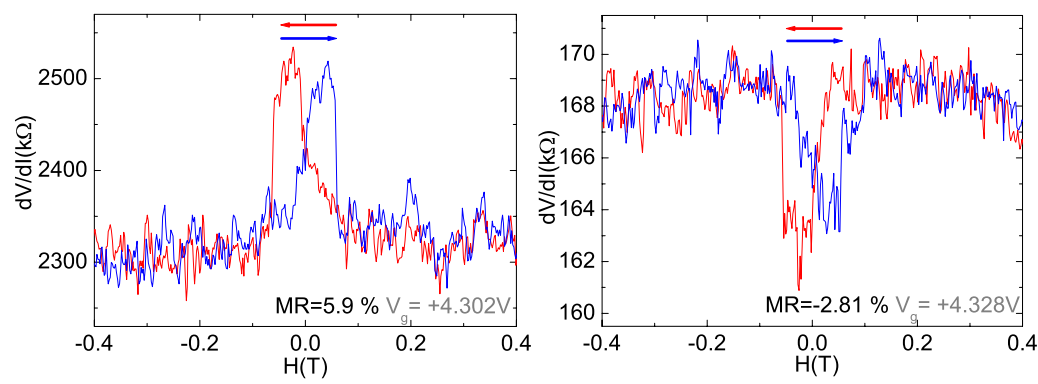

Figure 10. Example of magnetoresistance curves for the SWNT measurements of [29]. Depending on the gate voltage, both signs of MR are observed. Left: the MR observed is positive $(\mathrm{MR}=5.89 \%)$, for a gate voltage $V_{\mathrm{g}}=4.302 \mathrm{~V}$. Right: the MR observed for the same device is negative (MR $=-2.81 \%$ ), for a gate voltage $V_{\mathrm{g}}=4.328 \mathrm{~V}$.

gate voltage, MR is either positive or negative, as shown in figure 10. The amplitude of the effect is about 5\% for MWNTs and SWNTs in the 'Fabry-Perot' regime. It grows to about $10-15 \%$ for SWNTs in the Coulomb blockade regime. In addition, the sign of MR can also be controlled by $V_{\text {sd }}$ in both types of nanotubes $[29,57]$.

4.1.5. Other types of contacts. So far, we have only considered metallic ferromagnetic contacts to carbon nanotubes. This choice is generally led by the simplicity of the combination of thin metallic film growth with standard e-beam lithography techniques. The two main drawbacks of these methods are the small spin polarizations of the electrodes and the possible conductivity mismatch between the metal and the carbon nanotube.

The latter issue may be solved if the ferromagnetic contacts are ferromagnetic semiconductors. Such a method has recently been used by Jensen et al who used (Ga, Mn)As ferromagnetic electrode [54]. In this study, the Curie temperature of the contacts is about $70 \mathrm{~K}$. Therefore, the contacts are ferromagnetic at temperatures relevant for quantum transport in carbon nanotubes, but they cannot be used for applications at room temperature. The maximum amplitude of MR observed at $300 \mathrm{mK}$ is however very large, about $150 \%$, and both signs of MR are observed. In addition, the sign and the amplitude of MR depend on $V_{\mathrm{g}}$ and $V_{\mathrm{sd}}$.

Hueso et al [60] have used LSMO to electrically inject and detect spins in MWNTs. These materials have a bulk spin polarization of about $100 \%$. A MR of $37 \%$ is observed at $5 \mathrm{~K}$, and the spin signal persists up to $100 \mathrm{~K}$. Nevertheless, this scheme seems to produce samples with a high two-probe resistance of about $1 \mathrm{M} \Omega$ at $300 \mathrm{~K}$.

\subsection{The effect of contacts not related to spin injection}

As we have seen in section 3, spin-polarized transport induces a MR effect for ferromagnetically contacted nanotubes. However, a spin-valve-like behaviour does not automatically imply that spin injection is actually taking place in the nanotube. This just means that the resistance depends on the relative directions of the magnetizations of the two ferromagnets. Although this can be enough for spintronic devices [61], from a fundamental point of view, it is essential to separate spin injection related phenomena from the others. In this section, we introduce MR effects not directly related to the existence of spin-polarized transport inside the nanotube.
4.2.1. Stray field effects. Ferromagnetic electrodes not only induce a spin-dependent scattering at their interface but also generate an external stray field which can be under certain circumstances of the order of a few $100 \mathrm{mT}$ [62]. Therefore, two ferromagnetic microstrips like those shown in figure 9 can in principle generate a local magnetic field $H_{\mathrm{loc}}$ which will switch hysteretically as the magnetizations switch. Since low-dimensional conductors are very often sensitive to external magnetic fields, it is possible for a MR to appear just because charges couple naturally to the vector potential (such a sensitivity is reflected in the conductance of nanotubes connected to non-magnetic leads). Importantly, if this mechanism is effective with two ferromagnetic contacts, it should also be significant if only one contact is ferromagnetic, in contrast to the spin injection case.

In practice, a MWNT with ferromagnetic contacts has indeed in general a finite background MR superimposed on the hysteretic part of the MR. The field dependence of the non-hysteretic part of the MR can be quantified by a sensitivity $S$ in $\% \mathrm{~T}^{-1}$ to the local magnetic field. Figure 11 shows typical examples of hysteretic and background MR for a MWNT with NiPd contacts, for different applied gate voltages $V_{\mathrm{g}}$ [29]. The sensitivity $S$ is of the order of $1 \% \mathrm{~T}^{-1}$ or less and can change sign for different $V_{\mathrm{g}}$. From this figure, one can calculate the local field change $\Delta H_{\text {loc }}$ required to obtain the observed hysteretic MR. For $V_{\mathrm{g}}=-3.1 \mathrm{~V}$, one finds $\Delta H_{\mathrm{loc}}=-2.9 / 0.2=-14.5 \mathrm{~T}$, which is negative and way beyond what can be obtained with microstrips. Furthermore, for $V_{\mathrm{g}}=-3.3 \mathrm{~V}$, one would need a positive $\Delta H_{\text {loc }}$, since both MR and $S$ have the same negative sign. Such a sign change of the local magnetic field produced by two metallic ferromagnets for different gate voltages can hardly be explained. Therefore, stray field effects are not dominant in the MR signal for this type of F-MWNT-F device. In addition, as one can see in figure $10, S$ is in general smaller for SWNTs [29, 56, 57]. One can conclude that stray field effects do not contribute substantially to MR observed in nanotubes, at least for the NiPd devices realized so far.

4.2.2. Magneto-Coulomb (MC) effects. The magnetic field used to control the magnetization of the ferromagnetic contacts can also induce a magnetoresistance effect, independently of any spin-polarized transport process. This so-called magneto-Coulomb (MC) effect [63] simply requires that the conductance of the nanotube depends on its gate voltage $V_{\mathrm{g}}$. The magnetic field shifts the energies of spins $\uparrow(\downarrow)$ inside 

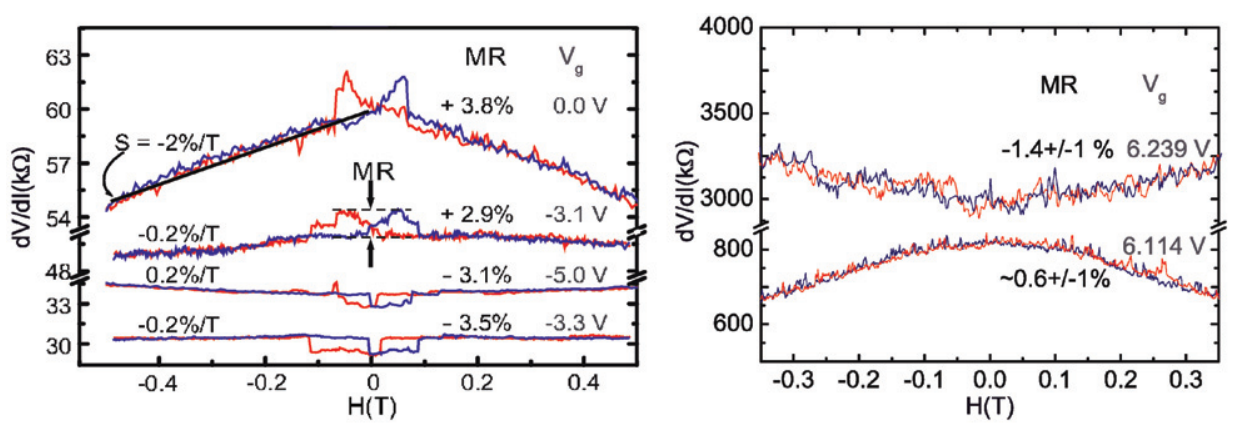

Figure 11. Left: MR observed by Sahoo et al [29] for a MWNT connected to two NiPd leads, with different values of $V_{\mathrm{g}}$. Depending on $V_{\mathrm{g}}$, both signs of MR and sensitivity $S$ are observed. The amplitude and the sign of $S$ are not correlated with MR. Therefore, the stray fields from the ferromagnetic electrodes cannot account for MR observed for this device. Right: resistance of a Pd-SWNT-PdNi device as a function of an external magnetic field for two values of $V_{\mathrm{g}}$. Almost no hysteresis is observed. The maximum amplitude can be estimated (almost within the noise) as $\Delta G / G \sim 1 \%$, more than an order of magnitude smaller than the observed signal with two NiPd electrodes.

contact $l$ by the Zeeman energy $\pm g \mu_{\mathrm{B}} H / 2$. Since the densities of states $N_{l, \sigma}=N_{l}\left(1+\sigma \eta_{l} p_{l}\right)$ at contacts $l \in\{\mathrm{L}, \mathrm{R}\}$ are spin dependent $\left(p_{l} \neq 0\right)$, this Zeeman shift must be compensated by a slight change $\Delta \mu_{l}=-p_{l} g \mu_{\mathrm{B}} H / 2$ in the Fermi energies of the contacts. This modifies the electrostatic profile of the circuit, leading to a conductance $\widetilde{G}\left(V_{\mathrm{g}}\right)=G\left(V_{\mathrm{g}}-C_{\mathrm{L}} \Delta \mu_{\mathrm{L}} / e\right.$ $\left.-C_{\mathrm{R}} \Delta \mu_{\mathrm{R}} / e\right)$. In this framework, a hysteretic conductance pattern can be obtained while sweeping the magnetic field to reverse the magnetization of the contacts. The amplitude of the magnetoresistance signal induced by the MC effect is [64]

$$
\mathrm{MR}=-\frac{1}{G} \frac{\mathrm{d} G}{\mathrm{~d} V_{\mathrm{g}}} \frac{g \mu_{\mathrm{B}}\left(p_{\mathrm{L}} C_{\mathrm{L}} H_{\mathrm{cL}}+p_{\mathrm{R}} C_{\mathrm{R}} H_{\mathrm{cR}}\right)}{e C_{\mathrm{g}}} .
$$

The expression of $G$ to insert in (11) depends on the different energy scales involved in the problem. For a device showing conductance peaks, the MC effect should induce a magnetoresistance effect with a sign oscillating with $V_{\mathrm{g}}$, since it is proportional to the derivative of $G\left(V_{\mathrm{g}}\right)$. Importantly, equation (11) shows that the MC-induced magnetoresistance effect should occur even in the presence of one single ferromagnetic contact. Finally, from the expression of $\widetilde{G}\left(V_{\mathrm{g}}\right)$, the MC effect produces a strong background variation in $G(H)$ on top of the discontinuities at $H=H_{\mathrm{cL}(\mathrm{R})}$ (see [64]). These features could be useful for an experimental identification of this effect.

4.2.3. Comparison between single and double ferromagnetic contacts schemes. Both the stray field effect mechanism and the MC effect should already be significant for devices with a single ferromagnetic contact if these effects are relevant with two ferromagnetic contacts. Therefore, it is useful to fabricate such devices and measure $\Delta G / G$. Such experiments have been carried out by Jensen et al with Fe-NT-Au contacts and no MR has ever been found [55]. With (Ga, Mn)As contacts, Jensen et al have reported a finite MR of about $10 \%$ for single ferromagnetic contacts, while the maximum amplitude for double ferromagnetic contacts is about $150 \%$. Figure 11, right, shows the $\Delta G / G$ measurement performed by [29] for a NiPd-SWNT-Pd device, for two different values of gate voltages, one in the Coulomb valley and the other close to a resonance. The upper bound for $\Delta G / G$ is $1.4 \%$ in amplitude which is one order of magnitude lower than the maximum $\Delta G / G$ observed with two ferromagnetic contacts, as can be seen in figure 14. Therefore, all the studies carried out so far point to the fact that contact effects are generally not dominant.

\section{Electric field control of spin transport}

In this section, we present the most advanced experimental results which have been reported so far about electric field control of spin-dependent transport in carbon nanotubes. All these experiments have been realized with NiPd contacts. For their interpretation, we focus on the Fabry-Perot and quantum dot regimes, which have been introduced theoretically in section 3 .

\subsection{Spectroscopy of carbon nanotubes with ferromagnetic contacts}

The spectroscopy of a carbon nanotube contacted to ferromagnetic leads can be realized by measuring its conductance as a function of the gate voltage $V_{\mathrm{g}}$ and the source-drain voltage $V_{\text {sd }}$. This step is essential to determine the different characteristic energies which set the behaviour of the nanotube and understand the physics leading to the MR effect. In the Fabry-Perot regime, the spectroscopy reveals the intrinsic energy spacing $\Delta E=h v_{\mathrm{Fw}} / 2 \ell$ of the quasi-bound states of the nanotube, where $\ell$ is the effective nanotube length on which transport is actually taking place (see figure 12, left panel). This length is generally defined by the inner spacing between the two metallic electrodes for SWNTs (see for instance [10]) but can also be related to the full tube length for MWNTs [65]. In the case of a quantum dot behaviour, the spectroscopy also reveals the charging energy $U=e^{2} / C_{\Sigma}$ of the nanotube device (see figure 14, left panel). In the latter case, other energy scales can be revealed in the fine structure of spectroscopy in metallic SWNTs [10], but we will omit them since they have not been identified in the F-SWNT-F experiments so far.

\subsection{Gate modulations of the magnetoresistance}

We have shown in section 3 that the MR of a quantum wire with ferromagnetic contacts can strongly depend on the gate voltage $V_{\mathrm{g}}$, in the Fabry-Perot regime as well as in the quantum dot regime. We show below how these phenomena can be revealed in ferromagnetically contacted MWNTs and SWNTs. 

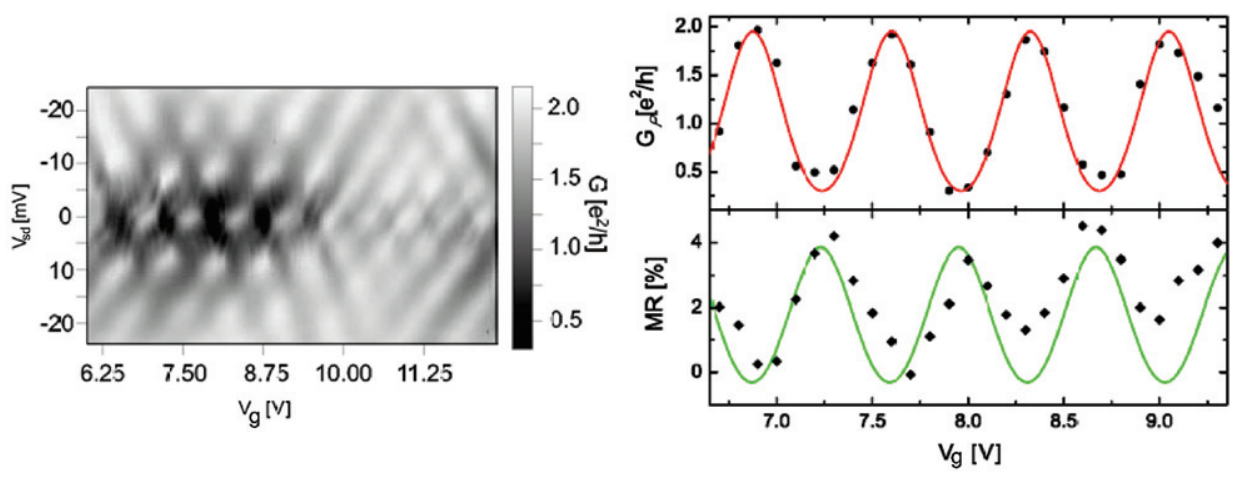

Figure 12. Experimental results obtained by [57] with a SWNT connected to two PdNi contacts. The left panel shows a colour (grey) scale plot of the nonlinear conductance $G^{\mathrm{P}}$ of the nanotube as a function of the source-drain voltage $V_{\text {sd }}$ and the gate voltage $V_{\mathrm{g}}$. The spacing between the resonant lines of conductance indicates an intrinsic energy spacing of the levels by $\Delta E \approx 7 \mathrm{meV}$. The right panel shows a comparison between the $G^{\mathrm{P}}\left(V_{\mathrm{g}}\right)$ and $\mathrm{MR}\left(V_{\mathrm{g}}\right)$ data measured at $T=4.2 \mathrm{~K}$ (symbols) and the non-interacting scattering theory of section 3.1.2, assuming two uncoupled channels with $T_{\mathrm{L}}=0.84, T_{\mathrm{R}}=0.26, P_{\mathrm{L}(\mathrm{R})}=0.1$ and no SDIPS.

\subsubsection{SWNTs in the Fabry-Perot regime. Man et al} [57] have measured MR for SWNTs with transparent NiPd contacts. In agreement with previous studies with nonferromagnetic contacts, the characteristic pattern of an electronic interferometer is observed, as shown in figure 12, left panel. The pattern reveals an intrinsic level spacing $\Delta E \sim 7 \mathrm{meV}$, which corresponds to zero-dimensional states delocalized over $300 \mathrm{~nm}$, in agreement with the lithographically defined SWNT length. Figure 12, right panel, displays simultaneous measurements of the MR and the linear conductance $G^{\mathrm{P}}$. The MR oscillates from $0 \%$ to $4 \%$ on the same gate scale as the linear conductance, e.g. $\Delta V_{\mathrm{g}} \approx 0.5 \mathrm{~V}$. Therefore, as anticipated from section 3.1, the MR can be gate controlled in SWNTs through quantum interferences.

In order to rule out possible contact effects, one can compare the experimental MR measured by [57] with the MR expected from the magneto-Coulomb effect. In principle, the so-called magneto-Coulomb (MC) effect can occur even in a non-interacting resonant wire since the conductance of the wire depends on its gate voltage $V_{\mathrm{g}}$. The amplitude expected for the MC-induced magnetoresistance, using equation (11) with $C_{\mathrm{g}} / C_{\Sigma}=0.014, G\left(\mathrm{~d} G / \mathrm{d} V_{\mathrm{g}}\right)^{-1}=0.125 \mathrm{~V}, H_{\mathrm{cL}(\mathrm{R})}<300 \mathrm{mT}$, $p_{\mathrm{L}(\mathrm{R})}=0.1$, is $|\mathrm{MR}|<0.2 \%$. This value is much weaker than the measured MR. Also, the experimental $\operatorname{MR}\left(V_{\mathrm{g}}\right)$ signal is clearly not proportional to the logarithmic derivative of $G\left(V_{\mathrm{g}}\right)$, in contrast to MR expected from equation (11). It is thus not possible to attribute the $\operatorname{MR}\left(V_{\mathrm{g}}\right)$ observed to the magnetoCoulomb effect introduced in section 4.2.2.

Resonant effects account much better for the observed MR. Indeed, Man et al [57] have interpreted their data with the model presented in section 3.1 (equations (1) and (2)). They have assumed two uncoupled identical channels in order to take into account the two-fold degeneracy commonly observed in SWNTs, with $T_{\mathrm{L}}=0.84, T_{\mathrm{R}}=0.26, P_{\mathrm{L}}=P_{\mathrm{R}}=0.1$ and no SDIPS. In view of the strong value of $T_{\mathrm{L}}+T_{\mathrm{R}}$ and of the low values of $P_{\mathrm{L}(\mathrm{R})}$, the effects of the SDIPS on the $\operatorname{MR}\left(V_{\mathrm{g}}\right)$ curves are indeed probably too weak to be resolved in the actual experiment. Nevertheless, it is interesting to note that the $\operatorname{MR}\left(V_{\mathrm{g}}\right)$ pattern of figure 12, right panel, shows a slightly asymmetric behaviour for $V_{\mathrm{g}}<7.7 \mathrm{~V}$, similar to the curve shown in the bottom-right panel of figure 4, plotted for $T_{\mathrm{L}}=0.84, T_{\mathrm{R}}=0.26, P_{\mathrm{L}}=P_{\mathrm{R}}=0.1$ and a finite SDIPS

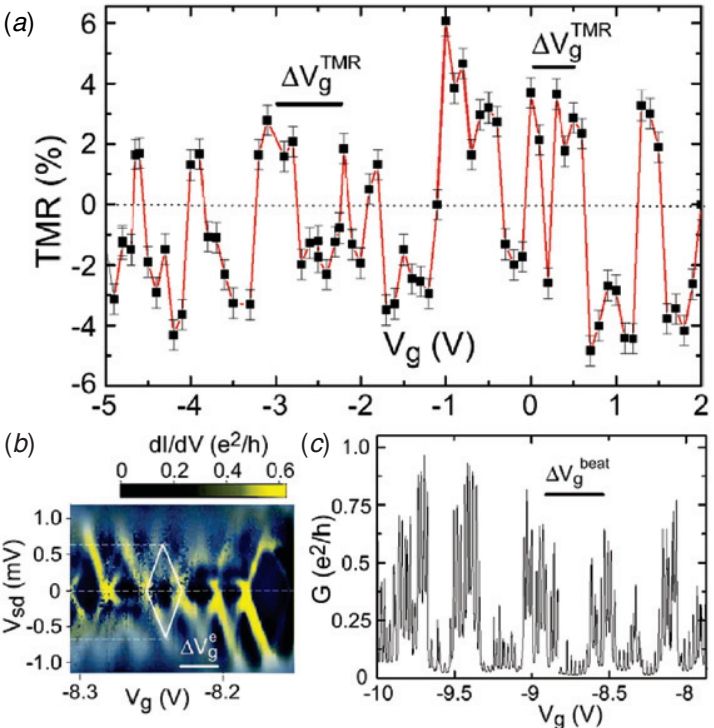

Figure 13. Experimental results obtained by [29] with a MWNT connected to two PdNi contacts. (a) The MR data measured at $T=1.85 \mathrm{~K}$. The MR oscillates with a period $\Delta V_{\mathrm{g}}^{\mathrm{TMR}} \sim 0.4-0.75 \mathrm{~V}$. (b) A colour (grey) scale plot of the nonlinear conductance $G^{\mathrm{P}}$ of the nanotube as a function of the source-drain voltage $V_{\mathrm{sd}}$ and the gate voltage $V_{\mathrm{g}}$, for $T=300 \mathrm{mK}$. This plot allows the resolution of the single-electron states, which correspond to a gate voltage scale $\Delta V_{\mathrm{g}}^{\mathrm{e}} \sim 25 \mathrm{mV}$, and indicate Coulomb blockade effects. (c) The conductance $G^{\mathrm{P}}$ of the device measured at $T=300 \mathrm{mK}$ on a $V_{\mathrm{g}}$ range much larger than that in (b). The conductance peaks show beatings with a period $\Delta V_{\mathrm{g}}^{\text {beat }} \sim 0.4 \mathrm{~V}$ comparable to $\Delta V_{\mathrm{g}}^{\mathrm{TMR}}$.

value $\Delta \varphi_{\mathrm{L}(\mathrm{R})}=-0.035$. The irregularities present in the variations of the $\operatorname{MR}\left(V_{\mathrm{g}}\right)$ data for $V_{\mathrm{g}}>7.7 \mathrm{~V}$ prevent a reliable conclusion on the presence of SDIPS in these data (the authors suggest that these irregularities are due to the misorientation of the magnetizations in the electrodes).

5.2.2. MWNTs. Sahoo et al [29] have studied the gate dependence of the MR for MWNTs with NiPd electrodes, at $T=1.85 \mathrm{~K}$. As shown in figure 13(a), the MR is observed to oscillate relatively regularly between $-5 \%$ and $+6 \%$ on a gate voltage scale $\Delta V_{\mathrm{g}}^{\mathrm{TMR}}$ such that $0.4 V<\Delta V_{\mathrm{g}}^{\mathrm{TMR}}<0.75 V$. 
The conductance of the same sample has been studied at lower temperatures $(T=300 \mathrm{mK})$, in order to resolve the single-electron states which could not be resolved at the temperature at which the MR was measured. A measurement of the differential conductance $\mathrm{d} I / \mathrm{d} V$ as a function of sourcedrain $V_{\mathrm{sd}}$ and gate voltage $V_{\mathrm{g}}$ at $T=300 \mathrm{mK}$ is shown in figure $13(b)$ for a relatively narrow $V_{\mathrm{g}}$ range. It displays the diamond-like pattern characteristic of single-electron tunnelling in a quantum dot. The diamonds vary in size with single-electron addition energies ranging between 0.5 and $0.75 \mathrm{meV}$, in agreement with previous reports on MWNT quantum dots with non-ferromagnetic leads [65]. The MR gate voltage scale $\Delta V_{\mathrm{g}}^{\mathrm{TMR}}$ measured at $T=1.85 \mathrm{~K}$ is much larger than the scale $\Delta V_{\mathrm{g}}^{\mathrm{e}} \sim 25 \mathrm{mV}$ for the addition of single electrons: it corresponds to the addition of at least 16 electrons rather than 1 .

In order to understand this discrepancy, one can consider the linear conductance observed over a wider gate voltage range, as shown in figure 13(c). The single-electron conductance peaks are strongly modulated in amplitude, leading to a regular beating pattern with a gate voltage scale $\Delta V_{\mathrm{g}}^{\text {beat }} \sim 0.4 \mathrm{~V}$. This scale corresponds to the scale $\Delta V_{\mathrm{g}}^{\mathrm{TMR}}$ of the MR oscillations, probably because, due to thermal averaging at $T=1.85 \mathrm{~K}$, the conductance is determined by the envelope of these beatings, which affects in turn the magnetoresistance.

Interestingly, such beatings can be found within the multichannel non-interacting picture introduced in section 3.1.3. In this model, at temperatures such that the single-particle resonances are averaged out, the MR is only sensitive to the average over these resonances, yielding a MR modulation that follows the envelope function of the singleelectron peaks (see figure 5).

5.2.3. SWNTs in the Coulomb blockade regime. Sahoo et al [29] have also studied the MR for SWNTs with NiPd contacts. Figure 14, left panel, displays a colour (grey scale) plot of the nonlinear conductance $\mathrm{d} I / \mathrm{d} V$ as a function of $V_{\mathrm{g}}$ and $V_{\mathrm{sd}}$ at $1.85 \mathrm{~K}$ for a SWNT device with NiPd electrodes. The characteristic quantum dot behaviour is observed. One has $E_{\mathrm{c}} \sim 5 \mathrm{meV}$ and $\Delta E \sim 2.5 \mathrm{meV}$. The latter value corresponds to zero-dimensional states delocalized on $\ell=600 \mathrm{~nm}$, in agreement with the lithographically defined SWNT length. In figure 14, right panel, the variations of the linear conductance $G$ and MR are simultaneously shown for two resonances. The MR changes sign on each conductance resonance. The amplitude of the MR ranges from $-7 \%$ to $+17 \%$, which is a higher amplitude than for the MWNT samples and SWNTs in the strongly coupled regime. Electron-electron interactions seem to enhance the amplitude of MR modulations, thereby improving the spin-FET behaviour.

In this paragraph, we compare the experimental MR reported in [29] with the MR expected from the magnetoCoulomb effect. One can evaluate the amplitude of the magnetoresistance induced by the $\mathrm{MC}$ effect in this experiment, using equation (11). With $C_{\Sigma} / C_{\mathrm{g}}=10$, $G\left(\mathrm{~d} G / \mathrm{d} V_{\mathrm{g}}\right)^{-1}=12.5 \mathrm{mV}, H_{\mathrm{cL}(\mathrm{R})}<100 \mathrm{mT}, p_{\mathrm{L}(\mathrm{R})}=0.4$, one obtains an amplitude $|\mathrm{MR}|<0.4 \%$, which is too weak to account for the data of figure 14. Also, the shape of the $\operatorname{MR}\left(V_{\mathrm{g}}\right)$ shown in this figure is clearly not proportional to the logarithmic derivative of $G\left(V_{\mathrm{g}}\right)$, contrarily to what is expected for the MC-induced magnetoresistance. Finally, circuits with a single ferromagnetic contact were also realized in order to check the origin of the MR effect observed. With a single ferromagnetic contact, the MR effect obtained (see figure 11) is much weaker, which rules out not only the MC effect but also stray field effects produced by the ferromagnetic leads. Therefore, one can consider the MR observed with two ferromagnetic contacts as an effect of spin injection in a resonant system.

In [29], Sahoo et al have used the scattering approach introduced in section 3.1 in order to interpret their data. The line shape of the MR dips is asymmetric, similar to the calculated line shape for the non-interacting regime displayed
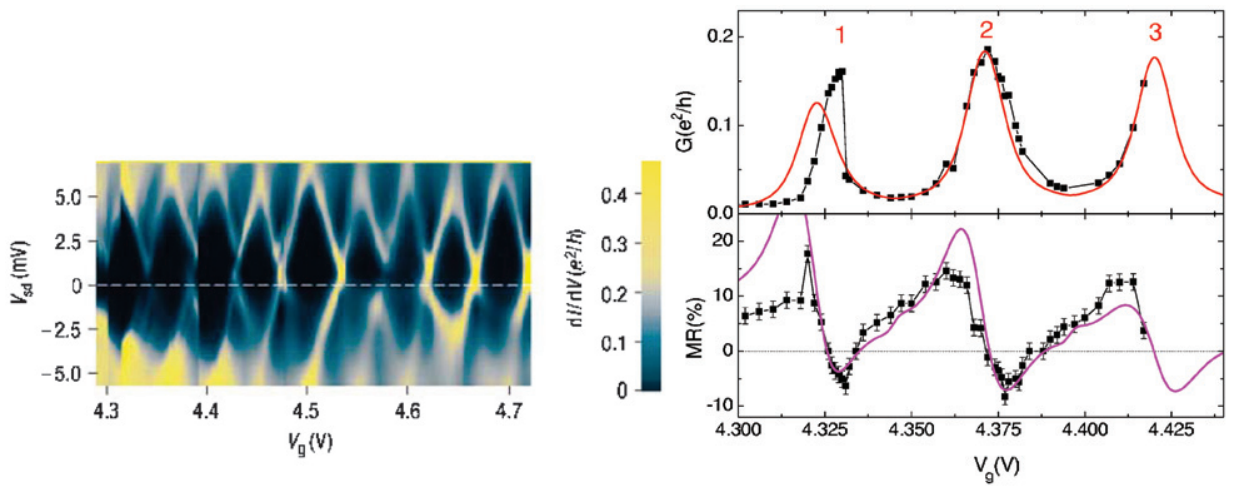

Figure 14. Experimental results obtained by [29] with a SWNT connected to two PdNi contacts. Left panel: colour (grey) scale plot of the nonlinear conductance $G^{\mathrm{P}}$ of the nanotube as a function of the source-drain voltage $V_{\text {sd }}$ and the gate voltage $V_{\mathrm{g}}$. This plot indicates an intrinsic energy spacing of the levels by $\Delta E=2.5 \mathrm{meV}$ and a charging energy $U=5 \mathrm{meV}$. Right panel: conductance $G^{\mathrm{P}}$ and magnetoresistance MR measured simultaneously at $T=1.85 \mathrm{~K}$ (black squares). As shown by [16], these curves can be interpreted in an interactive picture by using the EOM approach presented in section 3.2 for a quantum dot with two degenerate energy levels. The theoretical curves are shown for parameters consistent with the experiment, i.e. $U=5 \mathrm{meV}, U / k_{\mathrm{B}} T=30, \alpha=0.0986$ and $P_{\mathrm{L}(\mathrm{R})}=0.4$. Assuming identical tunnel couplings for the two orbitals, the values of tunnel rates $\Gamma_{\mathrm{L}}=0.0043 U$ and $\Gamma_{\mathrm{R}}=0.0725 U$ are imposed by the width and height of the conductance peaks. Then, $h_{\mathrm{SDIPS}}^{\mathrm{P} A \mathrm{P}]}$ are the only truly free fitting parameters remaining for interpreting the MR curve. The theory (coloured (full) lines) is plotted here for $g \mu_{\mathrm{B}} h_{\mathrm{SDIPS}}^{\mathrm{P}}=0.05 U$ and $h_{\mathrm{SDIPS}}^{\mathrm{AP}}=0$. 
in tigure 4, lett panel. 'This suggests that a tinite SDIPS can be observed in this circuit. Nevertheless, an interacting approach which takes into account the Coulomb blockade is required in order to confirm this point. We discuss below a fully interacting approach which allows a quantitative fit of the data, as shown in figure 14, right panel.

Reference [16] has provided an interactive interpretation of the data, using the EOM approach presented in section 3.2. The regularity of the $\operatorname{MR}\left(V_{\mathrm{g}}\right)$ oscillations displayed by the data being incompatible with a one-orbital model, a twodegenerate-orbital model, which takes into account the $K-K^{\prime}$ degeneracy of the orbital levels of the nanotube, has to be used. The two-orbital model exhibits good agreement with the experimental data for $h_{\mathrm{SDIPS}}^{\mathrm{P}}=0.05 U, h_{\mathrm{SDIPS}}^{\mathrm{AP}}=0, \Gamma_{\mathrm{L}} / U=$ $0.0043, \Gamma_{\mathrm{R}} / U=0.0725,\left|P_{\mathrm{L}(\mathrm{R})}\right|=0.4, U=5 \mathrm{meV}$, $U / k_{\mathrm{B}} T=30$ and $\alpha=0.0986$. Note that the two-orbital model could not provide a reasonable fit to the data for $h_{\mathrm{SDIPS}}^{\mathrm{P}[\mathrm{AP}]}=0$.

The value of $h_{\text {SDIPS }}^{\mathrm{P}}$ for the best fit corresponds to a magnetic field of about $2 \mathrm{~T}$, which is too strong to be attributed to stray fields from the ferromagnetic electrodes (see section 4.2.1). For comparison, one can estimate $h_{\text {SDIPS }}^{\mathrm{P}}$ in the non-interacting theory [18], using realistic parameters, i.e. leads with a Fermi energy $10 \mathrm{eV}$ and a density of states polarized by $40 \%$, and a nanotube with Fermi wave vector $8.5 \times 10^{9} \mathrm{~m}^{-1}$, Fermi velocity [8] $v_{\mathrm{Fw}}=8 \times 10^{5}$ $\mathrm{m} \mathrm{s}^{-1}$, length $\ell=500 \mathrm{~nm}$ as in [29], and density of states $N_{\mathrm{Fw}}=2 \ell / \pi \hbar v_{\mathrm{Fw}}$. The interfaces between the nanotube and the leads are furthermore modelled with Dirac potential barriers, with a height which is spin polarized by $40 \%$ and an average value which corresponds to (see section 3.2) $\Gamma_{\mathrm{L}(\mathrm{R})}=T_{\mathrm{L}(\mathrm{R})} / 2 \pi N_{\mathrm{F}}^{\mathrm{L}(\mathrm{R})} \sim 60 \mu \mathrm{eV}$ (for comparison the fitting parameters used in figure 14 correspond to $\Gamma_{\mathrm{L}}=21 \mu \mathrm{eV}$ and $\Gamma_{\mathrm{R}}=362 \mu \mathrm{eV}$ ). This gives $h_{\mathrm{SDIPS}}^{\mathrm{P}} \sim 1.3 \mathrm{~T}$, which is consistent with the above value used for the fit.

Note that the fitting curves shown in figure 14 have been optimized in order to interpret the data for $V_{\mathrm{g}}>4.331 \mathrm{~V}$. Like many Coulomb blockade devices, the nanotube circuit studied in this experiment suffered from low-frequency $V_{\mathrm{g}}$ noise, which can be attributed to charge fluctuators located in the vicinity of the device. At $V_{\mathrm{g}}=4.331 \mathrm{~V}$, a gate voltage jump occurred. Therefore, one cannot be sure that the data for $V_{\mathrm{g}}>4.331 \mathrm{~V}$ and $V_{\mathrm{g}}<4.331 \mathrm{~V}$ correspond to the filling of consecutive levels. Nevertheless, there is a certain probability that this is the case since these gate voltage jumps often have an amplitude which does not exceed $e / C_{\mathrm{g}}$. In this case, the discrepancy between the theory and the data could be due to the presence of other levels which should modify the theory for peak 1. In future experiments, it would be interesting to obtain continuous data on a larger $V_{\mathrm{g}}$ range, in order to check that the shape of the $\operatorname{MR}\left(V_{\mathrm{g}}\right)$ pattern depends on the occupation of the dot (a different shape is expected for peak 4 in the theory of [16]).

\subsection{Effect of source-drain bias on the magnetoresistance}

The effect of source-drain bias $V_{\text {sd }}$ on the MR can also be investigated in order to obtain a further understanding of the system. The MR at finite bias can be defined as $\left(\mathrm{d} V / \mathrm{d} I_{\mathrm{AP}}-\mathrm{d} V / \mathrm{d} I_{\mathrm{P}}\right) /\left(\mathrm{d} V / \mathrm{d} I_{\mathrm{P}}\right)$.
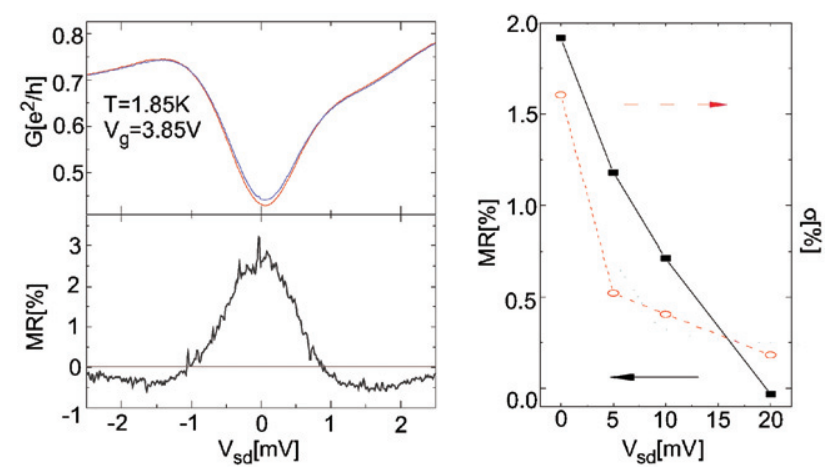

Figure 15. Effect of a finite source-drain voltage $V_{\text {sd }}$ on the magnetoresistance of a carbon nanotube. The left panel corresponds to a MWNT study by Sahoo [66]. The MR is displayed below the corresponding conductances in the AP (red full line) and the $\mathrm{P}$ orientations (blue full line), for a finite gate voltage of $3.85 \mathrm{~V}$. The right panel corresponds to the SWNT study of Man et al [57].

The MR corresponds here to a gate-voltage-averaged MR and $\sigma$ is the corresponding standard deviation. In both cases, the MR signal vanishes when the source-drain voltage increases.

Figure 15 displays two examples of MR as a function of $V_{\text {sd }}$. The left panel shows a measurement by Sahoo [66] of a MWNT with NiPd contacts separated by $1 \mu \mathrm{m}$ (this is a different MWNT sample than that discussed in section 5.2.2). The MR, which is about $3 \%$ at zero bias, gradually decreases at finite bias and vanishes for $V_{\mathrm{sd}}>3 \mathrm{mV}$. It displays a sign change, symmetrically for $\left|V_{\mathrm{sd}}\right|=1 \mathrm{mV}$. This energy scale corresponds to the zero-bias anomaly (ZBA) observed in the conductance data shown above MR. This ZBA has been reported in MWNTs and SWNTs. In the latter case, it has been attributed to the Luttinger liquid behaviour [36]. In MWNTs, the ZBA has been attributed to the interplay of electron-electron interactions and disorder [68]. Figure 15, right panel, shows the gate-voltage-averaged MR of a SWNT measured by Man et al [57] (this is the sample introduced in section 5.2.1). A similar trend as for MWNTs is observed.

Within the non-interacting picture, MR should display a similar dependence versus finite bias as versus gate voltage. However, the general features found experimentally contradict this simple assumption. The discrepancy between the noninteracting model and the data of figure 15 might therefore be due to interactions. In the interacting case, the Coulomb blockade can induce a non-trivial dependence of MR versus $V_{\text {sd }}$ [23]. For instance, dynamical spin blockade is expected to strongly affect spin transport at finite bias in quantum dots in the sequential tunnelling regime [28]. Non-trivial variations of $\operatorname{MR}\left(V_{\mathrm{sd}}\right)$ are also expected in the co-tunnelling regime [24]. Furthermore, oscillations of the MR signal are predicted in the Luttinger liquid limit [17]. However, the fact that MR does not seem to saturate to its classical value (i.e. that for two-tunnel junctions in series) but rather vanishes at high bias points to possible spin relaxation processes in the nanotube or to a bias dependence of the spin polarization in the electrodes [57].

\subsection{Spin relaxation time}

We have reported above on various experiments which indicate that a carbon nanotube can convey a spin-polarized current. The success of these experiments relies on the fact that 
electrons have a sutficiently long spin relaxation time $\tau_{\mathrm{s}}$ inside the nanotube. Nevertheless, for realizing further control of the spin dynamics in nanotubes, a detailed study of spin relaxation processes would be useful.

On the theoretical side, a few predictions for the value of $\tau_{\mathrm{s}}$ in carbon nanotubes are available [72]. In thin films made out of usual metals such as $\mathrm{Cu}$, one finds $\tau_{\mathrm{s}} \sim 10 \mathrm{ps}$ at low temperatures [70] due to mechanisms involving spinorbit coupling and momentum scattering [39]. In principle, one can expect a much larger $\tau_{\mathrm{s}}$ in nanotubes, due to the very weak spin-orbit interaction expected $[21,73]$ and the possible ballistic transport in these systems. Electronic confinement should further suppress conventional spin relaxation processes, as shown by [71] for GaAs quantum dots. One can thus expect that the dominant intrinsic relaxation mechanism arises from hyperfine coupling to the nuclear spins [39]. Nevertheless, the latter mechanism may not be so critical since ${ }^{12} \mathrm{C}$ does not have a nuclear spin and ${ }^{13} \mathrm{C}$, which has a nuclear spin $I=1 / 2$, has a low natural abundance of $1.1 \%$. Very recently, Semenov et al estimated $\tau_{\mathrm{s}} \sim 1 \mathrm{~s}$ due to the hyperfine interaction, for semiconducting SWNTs at $T=4 \mathrm{~K}$ [72].

On the experimental side, two different types of methods can be used to measure $\tau_{\mathrm{s}}$ in nanotubes: spin injection methods and spectral methods. Regarding spin injection, spin must be conserved for at least the dwell time of the electron on the nanotube in order to produce a finite spin signal in the conductance of the whole device. This allows us to estimate a lower bound for $\tau_{\mathrm{s}}$ from the experiments reported in this paper. From the measurements on SWNTs with NiPd contact in the weakly coupled regime [29], one finds $\tau_{\mathrm{S}}>2 \hbar /\left(\Gamma_{\mathrm{L}}+\Gamma_{\mathrm{R}}\right) \sim$ 3 ps at $T=1.85 \mathrm{~K}$. Regarding spectral methods, conduction electron spin resonance (CESR) has been used to investigate the spin relaxation processes in macroscopic amounts of carbon nanotubes [74, 75]. So far, no consensus has emerged from these measurements, especially concerning chemically undoped SWNTs. However, in all these experiments, it is found that magnetic impurities (probably catalytic particles) dominate the signal in general for unpurified nanotubes. For vacuum-annealed SWNTs, Petit et al could restore a finite CESR signal and determine a relatively long $\tau_{\mathrm{s}}$ of 3-5 ns at $T=300 \mathrm{~K}$. Nevertheless, this result has not been reproduced in later experiments [75]. Further research on spin relaxation mechanisms in nanotubes is highly desirable.

\section{Conclusion and perspectives}

In this paper, we have shown that carbon nanotubes are promising candidates for the realization of efficient spin transistors. Ferromagnetic contacts can be used to inject a spin-polarized current inside a nanotube, allowing the observation of spin-valve behaviour. Gate tunability of the nanotube magnetoresistance has been observed, in agreement with theoretical predictions made for resonant tunnelling systems and quantum dots.

From a technical point of view, the presently most advanced experiments regarding gate control of the magnetoresistance are not those which show the most efficient spin injection. An optimization of the contact properties has still to be done in this kind of experiment, in order to obtain accurate gate control of the giant magnetoresistance effect.
Experiments with highly polarized ferromagnetic materials should be further developed in order to increase the efficiency of spin injection and thus the amplitude of the MR effect. Another possibility to investigate is using ferromagnetic insulators as tunnel barriers. The shape of the ferromagnetic contacts should also be optimized in order to get better control of the switching behaviour of the magnetic polarizations. Another problem is the low temperatures required in order to obtain discrete levels on the nanotubes. In order to increase the operating temperature of the carbon-nanotube-based spinFET, one could reduce the spacing between the ferromagnetic electrodes down to a few $10 \mathrm{~nm}$, as suggested very recently [76].

From a fundamental point of view, a more extensive study of the dependence of the nanotube magnetoresistance on the gate voltage, source-drain voltage and temperature would allow the understanding of the physics involved to be refined. For instance, it would be interesting to investigate the effects of the gate voltage on the contacts' scattering properties. It would also be interesting to study how the spin dependence of interfacial phase shifts varies with the polarization of the contacts. Nanotube spin valves could also be used in non-collinear configurations in order to study spinprecession effects. Eventually, the relevant spin relaxation mechanisms should be identified.

The studies introduced in this paper open a path to the control and the manipulation of spin in nanotubes. Besides spintronics applications, we believe that devices such as those depicted here could also prove to be useful for quantum computing applications.

\section{Acknowledgments}

AC and TK acknowledge fruitful discussions with G Bauer, R Egger, A Fert, H Jaffrès, P Señeor, C Strunk and H S J van der Zant. HTM and AFM gratefully acknowledge FOM and NWO (Vernieuwingsimpuls 2000 program) for financial support. M-SC is supported by the SRC/ERC program (R11-2000-071) and the KFR grant (KFR-2005-070-C00055). This work was financially supported by the RTN Spintronics, DIENOW, by the Swiss NSF and the NCCR Nanoscience. AC is financially supported by Région Ile-de-France.

\section{References}

[1] Prinz G A 1998 Science 2821660

[2] Datta S and Das B 1990 Appl. Phys. Lett. 56665

[3] Schäpers Th, Nitta J, Heersche H B and Takayanagi H 2001 Phys. Rev. B 64125314

[4] Baibich M N, Broto J M, Fert A, Nguyen van Dau F and Petroff F 1988 Phys. Rev. Lett. 612472

[5] Binasch G, Grünberg P, Saurenbach F and Zinn W 1989 Phys. Rev. B 394828

[6] Julliere M 1975 Phys. Lett. A 54225

[7] Tans S J, Devoret M H, Groeneveld J A and Dekker C 1998 Nature 394761

[8] Liang W, Bockrath M, Bozovic D, Hafner J H, Tinkham M and Park H 2001 Nature 411665

[9] Blanter Ya M and Büttiker M 2000 Phys. Rep. 3361

[10] Sapmaz S, Jarillo-Herrero P, Kong J, Dekker C, Kouwenhoven L P and van der Zant H S J 2005 Phys. Rev. B 71153402

[11] Liang W, Bockrath M and Park H 2002 Phys. Rev. Lett. 88126801 
Babic B and Schönenberger C 2004 Phys. Rev. B 70195408 Jarillo-Herrero P, Kong J, van der Zant H S J, Dekker C, Kouwenhoven L P and De Franceschi S 2005 Phys. Rev. Lett. 94156802

Moriyama S, Fuse T, Suzuki M, Aoyagi Y and Ishibashi K 2005 Phys. Rev. Lett. 94186806

[12] Tsymbal E Y, Sokolov A, Sabirianov I F and Doudin B 2003 Phys. Rev. Lett. 90186602

[13] Brataas A, Nazarov Yu V and Bauer G E W 2000 Phys. Rev. Lett. 842481

Hernando D H, Nazarov Yu V, Brataas A and Bauer G E W 2000 Phys. Rev. B 625700

Brataas A, Nazarov Y V and Bauer G E W 2001 Eur. Phys. J. B 2299

[14] Tokuyasu T, Sauls J A and Rainer D 1988 Phys. Rev. B 388823

Millis A, Rainer D and Sauls J A 1988 Phys. Rev. B 384504

Fogelström M 2000 Phys. Rev. B 6211812

Cuevas J C and Fogelström M 2001 Phys. Rev. B 64104502

Chtchelkatchev N M, Belzig W, Nazarov Yu V and Bruder C 2001 JETP Lett. 74323

Huertas-Hernando D, Nazarov Yu V and Belzig W 2002 Phys. Rev. Lett. 88047003

Kopu J, Eschrig M, Cuevas J C and Fogelström M 2004 Phys. Rev. B 69094501

Zhao E, Löfwander T and Sauls J A 2004 Phys. Rev. B 70134510

Cottet A and Belzig W 2005 Phys. Rev. B 72180503

[15] Wetzels W, Bauer G E W and Grifoni M 2005 Phys. Rev. B 72020407

[16] Cottet A and Choi M-S 2006 Preprint cond-mat/0605264

[17] Balents L and Egger R 2000 Phys. Rev. Lett. 853464 Balents L and Egger R 2001 Phys. Rev. B 64035310

[18] Cottet A, Kontos T, Belzig W, Schönenberger C and Bruder C 2006 Europhys. Lett. $\mathbf{7 4} 320$

[19] Bachtold A, Strunk C, Salvetat J-P, Bonard J-M, Forro L Nussbaumer T and Schönenberger C 1999 Nature 397673

Bourlon B, Miko C, Forro L, Glattli D C and Bachtold A 2004 Phys. Rev. Lett. 93176806

[20] Saito R, Fujita M, Dresselhaus G and Dresselhaus M S 1992 Appl. Phys. Lett. 602204

[21] De Martino A and Egger R 2005 J. Phys.: Condens. Matter 175523

[22] Krüger M, Buitelaar M R, Nussbaumer T and Schönenberger C 2001 Appl. Phys. Lett. 781291

Krüger M, Widmer I, Nussbaumer T, Buitelaar M and Schönenberger C 2003 New J. Phys. 5138

[23] Barnas J and Fert A 1998 Phys. Rev. Lett. 801058

Braatas A, Nazarov Yu V, Inoue J and Bauer G E W 1999 Phys. Rev. B 5993

Imamura H, Takahashi S and Maekawa S 1999 Phys. Rev. B 596017

[24] Weymann I, König J, Martinek J, Barnas J and Schön G 2005 Phys. Rev. B 72115334

[25] König J and Martinek J 2003 Phys. Rev. Lett. 90166602

Braun M, König J and Martinek J 2004 Phys. Rev. B 70195345

König J, Martinek J, Barnas J and Schön G 2005 CFN Lectures on Functional Nanostructures (Lecture Notes in Physics vol 658) ed K Busch et al (Berlin: Springer) p 145

[26] Sergueev N, Sun Q-F, Guo H, Wang B G and Wang J 2002 Phys. Rev. B 65165303

Zhang P, Xue Q-K, Wang Y and Xie X C 2002 Phys. Rev. Lett. 89286803

López R and Sánchez D 2003 Phys. Rev. Lett. 90116602

Gazza C J, Torio M E and Riera J A 2006 Phys. Rev. B 73193108

[27] Martinek J, Utsumi Y, Imamura H, Barnas J, Maekawa S, König J and Schön G 2003 Phys. Rev. Lett. 91127203

Martinek J, Sindel M, Borda L, Barnas J, König J, Schön G and von Delft J 2003 Phys. Rev. Lett. 91247202
Choi M-S, Sánchez D and López R 2004 Phys. Rev. Lett. 92056601

Utsumi Y, Martinek J, Schön G, Imamura H and Maekawa S 2005 Phys. Rev. B 71245116

Martinek J, Sindel M, Borda L, Barnas J, Bulla R, König J, Schön G, Maekawa S and von Delft J 2005 Phys. Rev. B 72121302

Swirkowicz R, Wilczynski M and Barnas J 2006 J. Phys.: Condens. Matter 182291

Swirkowicz R, Wilczynski M, Wawrzyniak M and Barnas J 2006 Phys. Rev. B 73193312

[28] Bulka B R 2000 Phys. Rev. B 621186

Cottet A, Belzig W and Bruder C 2004 Phys. Rev. Lett. 92206801

Cottet A, Belzig W and Bruder C 2004 Phys. Rev. B 70115315

[29] Sahoo S, Kontos T, Furer J, Hoffmann C, Gräber M, Cottet A and Schönenberger C 2005 Nat. Phys. 199

[30] Meir Y and Wingreen N S 1992 Phys. Rev. Lett. 682512

[31] Meir Y, Wingreen N S and Lee P A 1991 Phys. Rev. Lett. 663048

[32] Aleiner I L, Brouwer P W and Glazman L I 2002 Phys. Rep. 358309

[33] Pasupathy A, Bialczak R C, Martinek J, Grose J E, Donev L A K, McEuen P L and Ralph D C 2004 Science 30686

[34] Nygard J, Cobden D H and Lindelof P E 2000 Nature 408342

Buitelaar M R, Bachtold A, Nussbaumer T, Iqbal M and Schönenberger C 2002 Phys. Rev. Lett. 88156801

Buitelaar M R, Nussbaumer T and Schönenberger C 2002 Phys. Rev. Lett. 89256801

Liang W, Bockrath M and Park H 2002 Phys. Rev. Lett. 88126801

Babic B, Kontos T and Schönenberger C 2004 Phys. Rev. B 70235419

[35] Yao Z, Postma H W Ch, Balents L and Dekker C 1999 Nature 402273

[36] Bockrath M, Cobden D H, Lu J, Rinzler A G, Smalley R E Balents L and McEuen P L 1999 Nature 397598

[37] Auslaender O M, Steinberg H, Yacoby A, Tserkovnyak Y, Halperin B I, Baldwin K W, Pfeiffer L N and West K W 2005 Science $\mathbf{3 0 8} 88$

[38] Peça C S and Balents L 2003 Phys. Rev. B 68205423

[39] Zutic I, Fabian J and Sarma S Das 2004 Rev. Mod. Phys. 76323

[40] Schmidt G, Ferrand D, Molenkamp L W, Filip A T and van Wees B J 2000 Phys. Rev. B 624790

[41] Rashba E I 2000 Phys. Rev. B 62 R16267

[42] Fert A and Jaffrès H 2001 Phys. Rev. B 64184420

[43] Hu C-M and Matsuyama T 1998 Phys. Rev. Lett. 87066803

[44] Hao X, Moodera J S and Meservey R 1990 Phys. Rev B 428235

Gajek M, Bibes M, Barthélémy A, Bouzehouane K, Fusil S, Varela M, Fontcuberta J and Fert A 2005 Phys. Rev. B 72020406

[45] Bezryadin A, Verschueren A R M, Tans S J and Dekker C 1998 Phys. Rev. Lett. 804036

[46] Gao B, Chen Y F, Fuhrer M S, Glattli D C and Bachtold A 2005 Phys. Rev. Lett. 95196802

[47] Kim J R, So H M, Kim J J and Kim J 2002 Phys. Rev. B 66233401

[48] Tombros N, van der Molen S J and van Wees B J 2006 Phys. Rev. B 73233403

[49] Tsukagoshi K, Alphenaar B W and Ago H 1999 Nature 401572

[50] Tsukagoshi K and Alphenaar B W 2000 Superlattices Microstruct. 27565

[51] Zhao B, Mönch I, Mühl T, Vinzelberg H and Schneider C M 2002 J. Appl. Phys. 917026

[52] Kong J, Franklin N R, Zhou C, Chapline M G, Peng S, Cho K and Dai H 2000 Science 287622 
Collıns P G, Bradley K, Ishıgamı M and Lettl A 2000 Science 2871801

Jhi S-H, Louie S G and Cohen M L 2000 Phys. Rev. Lett. 851710

[53] Kim W, Javey A, Vermesh O, Wang Q, Li Y and Dai H 2003 Nano Lett. 3193

[54] Jensen A, Hauptmann J R, Nygârd J and Lindelof P E 2005 Phys. Rev. B 72035419

[55] Jensen A 2003 PhD Thesis Technical University of Denmark, Copenhagen

[56] Nagabhirava B, Bansal T, Sumanasekera G U, Alphenaar B W and Liu L 2006 Appl. Phys. Lett. 88023503

[57] Man H T, Wever I J W and Morpurgo A F 2006 Phys. Rev. B 73241401

[58] Sahoo S, Kontos T, Schönenberger C and Suergers C 2005 Appl. Phys. Lett. 86112109

[59] Javey A, Guo J, Wang Q, Lundstrom M and Dai H 2003 Nature 424654

see also Babic B, Kontos T and Schönenberger C 2004 Phys Rev. B 70235419

[60] Hueso L E, Pruneda J M, Ferrari V, Burnell G, Valdés-Herrera J P, Simmons B D, Littlewood P B, Artacho E and Mathur N D 2005 Preprint cond-mat/0511697

[61] Wunderlich J et al 2006 Phys. Rev. Lett. 97077201 (Preprint cond-mat/0602608)

[62] Meier L, Salis G, Ellenberger C, Ensslin K and Gini E 2006 Appl. Phys. Lett. 88172501

[63] Shimada H, Ono K and Ootuka Y 1997 J. Phys. Soc. Japan 671359
Uno K, Shımada H and Uotuka Y 1998 J. Phys. Soc. Japan 672852

[64] van der Molen S J, Tombros N and van Wees B J 2006 Phys. Rev. B 73220406

[65] Buitelaar M R, Bachtold A, Nussbaumer T, Iqbal M and Schönenberger C 2002 Phys. Rev. Lett. 88156801

[66] Sahoo S 2005 PhD Thesis University of Basel, Basel

[67] Jedema F J, Heersche H B, Filip A T, Baselmans J J A and van Wees B J 2002 Nature 416713

[68] Bachtold A, de Jonge M, Grove-Rasmussen K, McEuen P L, Buitelaar M and Schönenberger C 2001 Phys. Rev. Lett. 87166801

[69] Beneu F and Monod P 1978 Phys. Rev. B 182422

[70] Jedema F J, Filip A T and van Wees B J 2001 Nature 410345

[71] Khaetskii A V and Nazarov Y V 2000 Phys. Rev. B 6112639

[72] Semenov Y G, Kim K W and Iafrate G J 2006 Preprint cond-mat 0602425

[73] Ando T 2000 J. Phys. Soc. Japan 691757

[74] Petit P, Jouguelet E, Fischer J E, Rinzler A G and Smalley R E 1997 Phys. Rev. B 569275

[75] Bandow S, Asaka S, Zhao X and Ando Y 1998 Appl. Phys. A 6723

Claye A S, Nemes N M, Jánossy A and Fischer J E 2000 Phys. Rev. B 62 R4845

Shen K, Tierney D L and Pietraß T 2003 Phys. Rev. B 68165418

Salvetat J-P, Fehér T, L'Huillier C, Beneu F and Forró L 2005 Phys. Rev. B 72075440

[76] Zutic I and Fuhrer M 2005 Nat. Phys. 185 